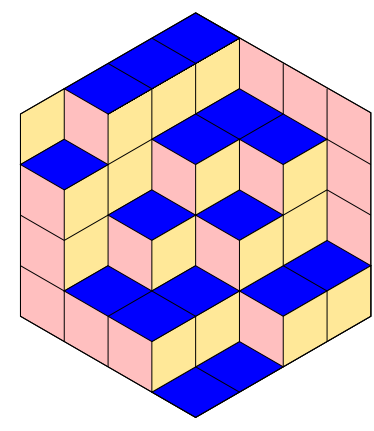

ALGEBRAIC COMBINATORICS

Henry Kvinge, Anthony M. Licata \& Stuart Mitchell

Khovanov's Heisenberg category, moments in free probability, and shifted symmetric functions

Volume 2, issue 1 (2019), p. 49-74.

<http://alco.centre-mersenne.org/item/ALCO_2019__2_1_49_0>

(C) The journal and the authors, 2019.

Some rights reserved.

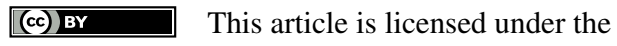

Creative Commons ATtribution 4.0 International License.

http://creativecommons.org/licenses/by/4.0/

Access to articles published by the journal Algebraic Combinatorics on the website http://alco.centre-mersenne.org/ implies agreement with the Terms of Use (http://alco.centre-mersenne.org/legal/).

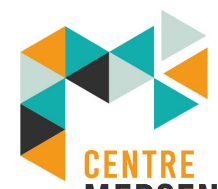

MERSENNE

Algebraic Combinatorics is member of the

Centre Mersenne for Open Scientific Publishing

www.centre-mersenne.org 


\title{
Khovanov's Heisenberg category, moments in free probability, and shifted symmetric functions
}

\author{
Henry Kvinge, Anthony M. Licata \& Stuart Mitchell
}

\begin{abstract}
We establish an isomorphism between the center End $_{\mathcal{H}^{\prime}}(\mathbb{1})$ of the Heisenberg category defined by Khovanov in [13] and the algebra $\Lambda^{*}$ of shifted symmetric functions defined by Okounkov-Olshanski in [18]. We give a graphical description of the shifted power and Schur bases of $\Lambda^{*}$ as elements of $\operatorname{End}_{\mathcal{H}^{\prime}}(\mathbb{1})$, and describe the curl generators of $\operatorname{End}_{\mathcal{H}^{\prime}}(\mathbb{1})$ in the language of shifted symmetric functions. This latter description makes use of the transition and co-transition measures of Kerov [10] and the noncommutative probability spaces of Biane [2]
\end{abstract}

\section{INTRODUCTION}

In [13], Khovanov introduces a graphical calculus of oriented planar diagrams and uses it to define a linear monoidal category $\mathcal{H}^{\prime}$, which he proposes as a categorification of the Heisenberg algebra. We denote by $\operatorname{End}_{\mathcal{H}^{\prime}}(\mathbb{1})$ the endomorphism algebra of the monoidal unit in $\mathcal{H}^{\prime}$. The commutative algebra $\operatorname{End}_{\mathcal{H}^{\prime}}(\mathbb{1})$ is, by definition, the algebra of closed oriented planar diagrams modulo the relations of the Khovanov graphical calculus. In his study of morphism spaces of $\mathcal{H}^{\prime}$, Khovanov introduces two sets of generators for $\operatorname{End}_{\mathcal{H}^{\prime}}(\mathbb{1})$ : the clockwise curls $\left\{c_{k}\right\}_{k \geqslant 0}$ and the counterclockwise curls $\left\{\tilde{c}_{k}\right\}_{k \geqslant 2}$. He then establishes algebra isomorphisms

$$
\operatorname{End}_{\mathcal{H}^{\prime}}(\mathbb{1}) \cong \mathbb{C}\left[c_{0}, c_{1}, c_{2}, \ldots\right] \cong \mathbb{C}\left[\tilde{c}_{2}, \tilde{c}_{3}, \tilde{c}_{4}, \ldots\right],
$$

and describes a recursion for expressing the clockwise and counterclockwise curls in terms of each other. He then relates $\mathcal{H}^{\prime}$ to representation theory by defining a sequence of functors $f_{k}^{\mathcal{H}^{\prime}}$ from $\mathcal{H}^{\prime}$ to bimodule categories for symmetric groups. A consequence of the existence of these functors is the existence of surjective algebra homomorphisms,

$$
f_{n}^{\mathcal{H}^{\prime}}: \operatorname{End}_{\mathcal{H}^{\prime}}(\mathbb{1}) \longrightarrow Z\left(\mathbb{C}\left[S_{n}\right]\right),
$$

from $\operatorname{End}_{\mathcal{H}^{\prime}}(\mathbb{1})$ to the center of the group algebra of each symmetric group. Based in part on this, Khovanov suggests that there should be a close connection between $\operatorname{End}_{\mathcal{H}^{\prime}}(\mathbb{1})$ and the asymptotic representation theory of symmetric groups. Furthermore, one might hope that $\operatorname{End}_{\mathcal{H}^{\prime}}(\mathbb{1})$ in fact gives a diagrammatic description of some algebra of pre-existing combinatorial interest.

Manuscript received 19th August 2017, revised 11th May 2018, accepted 9th July 2018.

KEYWORDS. Symmetric functions, asymptotic representation theory, Heisenberg categorification, graphical calculus.

ACKnowledgements. AML was supported by a Discovery Project grant from the Australian Research Council. 
The main goal of the current paper is to make precise the connection between $\operatorname{End}_{\mathcal{H}^{\prime}}(\mathbb{1})$ and both the asymptotic representation theory of symmetric groups and algebraic combinatorics. We do this by establishing an isomorphism between

$$
\varphi: \operatorname{End}_{\mathcal{H}^{\prime}}(\mathbb{1}) \longrightarrow \Lambda^{*},
$$

where $\Lambda^{*}$ is the shifted symmetric functions of Okounkov-Olshanski [18]. (See Theorem 5.3.) The algebra of shifted symmetric functions $\Lambda^{*}$ is a deformation of the algebra of symmetric functions. As is the case for $\operatorname{End}_{\mathcal{H}^{\prime}}(\mathbb{1})$, there are surjective algebra homomorphisms

$$
f_{n}^{\Lambda^{*}}: \Lambda^{*} \longrightarrow Z\left(\mathbb{C}\left[S_{n}\right]\right)
$$

to the center of the group algebra of each symmetric group. The isomorphism $\varphi$ : $\operatorname{End}_{\mathcal{H}^{\prime}}(\mathbb{1}) \longrightarrow \Lambda^{*}$ is canonical, in that it intertwines the homomorphisms $f_{n}^{\mathcal{H}^{\prime}}$ and $f_{n}^{\Lambda^{*}}$.

The isomorphism $\varphi: \operatorname{End}_{\mathcal{H}^{\prime}}(\mathbb{1}) \longrightarrow \Lambda^{*}$ allows us to give a graphical description of several important bases of $\Lambda^{*}$. For example, the shifted power sum denoted $p_{\lambda}^{\#}$ in [18] appears in $\operatorname{End}_{\mathcal{H}^{\prime}}(\mathbb{1})$ as the closure of a permutation of cycle type $\lambda$. The shifted Schur function $s_{\lambda}^{*}$ appears as the closure of a Young symmetrizer of type $\lambda$. (See Theorem 5.4).

In the other direction, it is also reasonable to ask for a description of the image of Khovanov's curl generators $c_{k}$ and $\tilde{c}_{k}$ as elements of $\Lambda^{*}$. It turns out that the right language for such a description is that of noncommutative probability theory. In [10], Kerov introduces, for each partition $\lambda$, a pair of finitely supported probability measures on $\mathbb{R}$; these probability measures are known as the transition and co-transition measures, or sometimes as growth and decay. In work of Biane [2], these probability measures appear as the compactly-supported measures associated to self-adjoint operators on a noncommutative probability space, and as a result they are basic objects of interest at the intersection of representation theory and noncommutative probability theory. In particular, the moments and Boolean cumulants of the transition and co-transition measures may be regarded as elements of $\Lambda^{*}$. In Theorem 5.5, we show that the isomorphism $\varphi$ takes Khovanov's curl generators $c_{k}$ and $\tilde{c}_{k}$ to scalar multiples of the $k$ th moments of Kerov's transition and co-transition measures. In fact, the close relationship between the transition and co-transition measures themselves yields two independent descriptions of the image of the curl generator $c_{k}$ : it is equal to a scalar multiple of both the $k$ th moment of the co-transition measure and the $(k+2)$ th Boolean cumulant of the transition measure. The observation that the Boolean cumulants of the transition measure are equal to the moments of the co-transition measure is closely connected to the adjointness of induction and restriction functors between representation categories of symmetric groups. A dictionary between several of the bases of $\operatorname{End}_{\mathcal{H}^{\prime}}(\mathbb{1})$ and $\Lambda^{*}$ is given in Table 1 below.

The existence of a relationship between $\mathcal{H}^{\prime}$ and free probability, and indeed, much of this paper, was anticipated by Khovanov in [13]. The relationship between generators of $\operatorname{End}_{\mathcal{H}^{\prime}}(\mathbb{1})$ and the noncommutative probability spaces of [2] may be seen as a further manifestation of the "planar structure" of free probability; the many connections between noncommutative probability and other mathematical subjects with planar structure are emphasized in the work of Guionnet, Jones and Shlyakhtenko [6].

In addition to the center of $\mathcal{H}^{\prime}$, another algebra of interest in the study of $\mathcal{H}^{\prime}$ is its trace (or zeroth Hochschild homology). The trace of $\mathcal{H}^{\prime}$ is an infinite-dimensional noncommutative algebra, which may be defined diagrammatically as the algebra of diagrams on an annulus; the trace acts naturally on $\operatorname{End}_{\mathcal{H}^{\prime}}(\mathbb{1})$ by gluing annular diagrams around planar ones. In [4], the trace of $\mathcal{H}^{\prime}$ is shown to be isomorphic to the $W_{1+\infty}$ algebra of conformal field theory. An action of $W_{1+\infty}$ on $\Lambda^{*}$ appears to be well known in the vertex algebra community, and such an action is constructed explicitly 


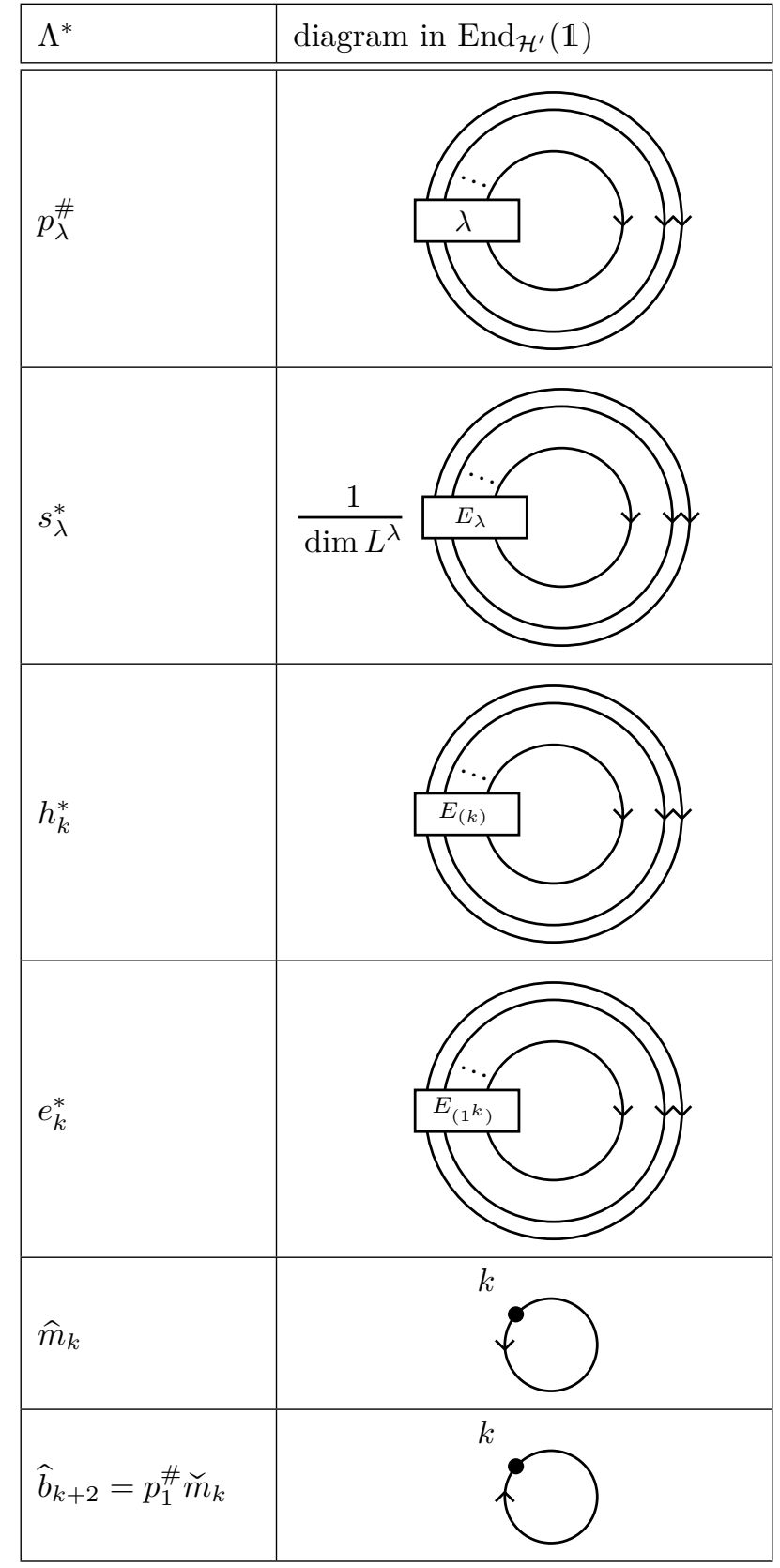

TABLE 1. A dictionary between $\Lambda^{*}$ and diagrams in $\operatorname{End}_{\mathcal{H}^{\prime}}(\mathbb{1})$.

in the work of Lascoux-Thibon [14]. Thus the isomorphism $\varphi: \operatorname{End}_{\mathcal{H}^{\prime}}(\mathbb{1}) \longrightarrow \Lambda^{*}$ of Theorem 5.3, together with the main result of [4], gives a purely planar realization, via Khovanov's graphical calculus, of Lascoux-Thibon's construction. 


\section{The SYMMETRIC GROUP AND ITS NORMALIZED CHARACTER THEORY}

We begin by establishing notation related to partitions and Young diagrams. Let $\mathcal{P}_{n}$ be the set of partitions of $n$ and

$$
\mathcal{P}:=\bigcup_{n \geqslant 0} \mathcal{P}_{n}
$$

For this section let $\lambda=\left(\lambda_{1}, \lambda_{2}, \ldots, \lambda_{r}\right) \in \mathcal{P}_{n}$ and $\mu=\left(\mu_{1}, \ldots, \mu_{t}\right) \in \mathcal{P}_{k}$ with $n \geqslant k$. We assume that $\lambda_{1} \geqslant \cdots \geqslant \lambda_{r}>0$ and $\mu_{1} \geqslant \cdots \geqslant \mu_{t}>0$. When $i>r$ (respectively $i>t$ ) we understand that $\lambda_{i}=0$ (resp. $\mu_{i}=0$ ). We use the following notation throughout:

- $n=\lambda_{1}+\lambda_{2}+\cdots+\lambda_{r}=:|\lambda|$.

- $\lambda \cup \mu$ is the partition formed from the union of the parts of $\lambda$ and $\mu$.

- $\mu \subseteq \lambda$ if $\mu_{i} \leqslant \lambda_{i}$ for all $i \geqslant 1$. When this is the case, we write $\lambda / \mu$ for the associated skew diagram.

- $\phi_{k, n}: \mathcal{P}_{k} \hookrightarrow \mathcal{P}_{n}$ is the function defined by $\phi_{k, n}(\mu)=\mu \cup 1^{n-k} \in \mathcal{P}_{n}$.

EXAMPLE 2.1. If $\mu=(3,2,1,1,1) \in \mathcal{P}_{8}$ then $\phi_{8,10}(\mu)=(3,2,1,1,1,1,1) \in \mathcal{P}_{10}$.

We freely identify $\mu \in \mathcal{P}$ with its corresponding Young diagram, which we draw using Russian notation (see Example 2.2). If $\square$ is a cell in the $i$ th row and $j$ th column of $\mu$ then the content of $\square$ is defined to be the integer

$$
\operatorname{cont}(\square):=j-i \text {. }
$$

We say that a cell $\square \notin \mu$ is $i$-addable with respect to $\mu$ if it has content $i$ and adding it to $\mu$ gives a Young diagram. We say that a cell $\square \in \mu$ is $i$-removable with respect to $\mu$ if it has content $i$ and removing it from $\mu$ gives a Young diagram. We call two sequences $a_{1}, \ldots, a_{d}$ and $b_{1}, \ldots, b_{d-1}$ interlacing when

$$
a_{1}<b_{1}<a_{2}<\cdots<a_{d-1}<b_{d-1}<a_{d} .
$$

The center of this pair of sequences is defined as the quantity $\left(a_{1}+\cdots+a_{d}\right)-\left(b_{1}+\right.$ $\left.\cdots+b_{d-1}\right)$. Each Young diagram $\mu$ uniquely defines two integer valued interlacing sequences $a_{1}, \ldots, a_{d}$ and $b_{1}, \ldots, b_{d-1}$ where:

- $a_{1}, \ldots, a_{d}$ is the ordered list of all $a_{j}$ such that there exists an $a_{j}$-addable cell with respect to $\mu$.

- $b_{1}, \ldots, b_{d-1}$ is the ordered list of all $b_{j}$ such that there exists a $b_{j}$-removable cell with respect to $\mu$.

From this description it is clear that $a_{1}, \ldots, a_{d}$ and $b_{1}, \ldots, b_{d-1}$ are interlacing.

EXAMPLE 2.2. Let $\mu=(4,2,1)$. Then $\mu$ yields the interlacing sequences

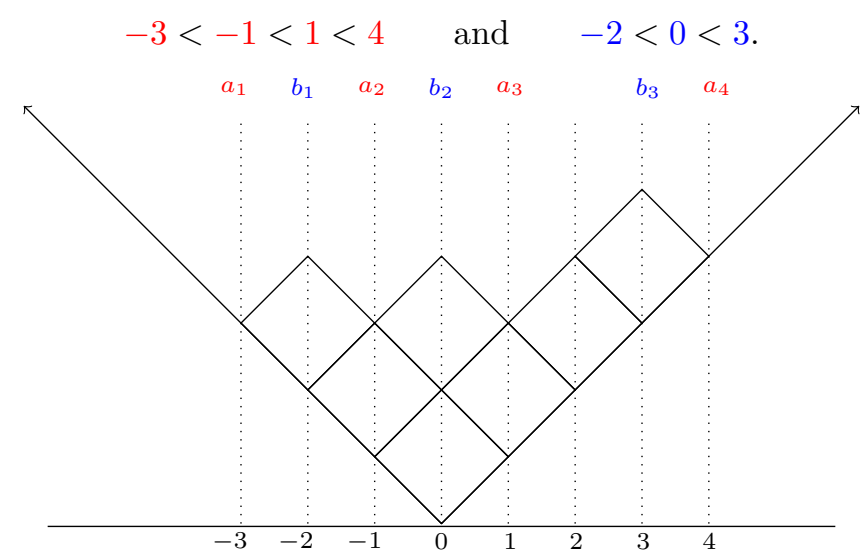


Proposition 2.3 ([11]). If $a_{1}, \ldots, a_{d}$ and $b_{1}, \ldots, b_{d-1}$ are the pair of interlacing sequences associated to a Young diagram then their center is 0 . Conversely, any pair of integer valued interlacing sequences with center 0 is associated to a Young diagram.

When $\mu \subseteq \lambda$ and $\lambda / \mu=\square$, then we write $\mu \nearrow \lambda$. In other words, $\mu \nearrow \lambda$ whenever we can obtain $\lambda$ from $\mu$ by adding a single cell. If $a_{1}, \ldots, a_{d}$ and $b_{1}, \ldots, b_{d-1}$ are the interlacing sequences associated to $\mu$, then we denote by $\mu^{(i)}$ the Young diagram that we get by adding a cell of content $a_{i}$, so that

$$
\operatorname{cont}\left(\mu^{(i)} / \mu\right)=a_{i}
$$

Similarly, we denote by $\mu_{(i)}$ the Young diagram that we get by removing a cell of content $b_{i}$ from $\mu$, so that

$$
\operatorname{cont}\left(\mu / \mu_{(i)}\right)=b_{i} .
$$

Note that $\mu_{(i)} \nearrow \mu$, while $\mu \nearrow \mu^{(i)}$.

ExAmple 2.4. If $\mu=(4,2,1)$ as in Example 2.2, we have

$$
\begin{aligned}
& \mu^{(1)}=(4,2,1,1) \\
& \mu^{(2)}=(4,2,2) \quad \mu_{(1)}=(4,2) \\
& \mu^{(3)}=(4,3,1) \quad \text { and } \quad \mu_{(2)}=(4,1,1) \\
& \mu^{(4)}=(5,2,1) \quad \mu_{(3)}=(3,2,1) .
\end{aligned}
$$

Let $S_{n}$ be the symmetric group. $S_{n}$ is generated by Coxeter generators $s_{1}, \ldots, s_{n-1}$ where $s_{i}$ is the adjacent transposition $(i, i+1)$. We identify $\mathbb{C}\left[S_{0}\right] \cong \mathbb{C}$. If $g \in S_{n}$ has cycle type $\lambda \vdash n$, then we write $\operatorname{sh}(g):=\lambda$. For $k \leqslant n$, there is an embedding $S_{k} \hookrightarrow S_{n}$ called the standard embedding which sends $S_{k}$ to the subgroup generated by $s_{1}, \ldots, s_{k-1}$, which stabilizes $\{k+1, \ldots, n\}$ pointwise. We extend this embedding by linearity to get an embedding of group algebras which we denote by $\iota_{k, n}: \mathbb{C}\left[S_{k}\right] \hookrightarrow$ $\mathbb{C}\left[S_{n}\right]$. We write $1_{k}$ for the identity element in $\mathbb{C}\left[S_{k}\right]$ so that $\iota_{k, n}\left(1_{k}\right)=1_{n}$. We write $w_{0, n}$ for the longest element of $S_{n}$ by Coxeter length. $w_{0, n}$ is the product of disjoint transpositions $(1, n),(2, n-1),(3, n-2), \ldots$ and in terms of Coxeter generators can be written

$$
w_{0, n}=\left(s_{1} s_{2} \ldots s_{n-1}\right)\left(s_{1} s_{2} \ldots s_{n-2}\right) \ldots\left(s_{1} s_{2}\right)\left(s_{1}\right) .
$$

For $\lambda \vdash n$, let $L^{\lambda}$ be the simple $\mathbb{C}\left[S_{n}\right]$-module (the irreducible $S_{n}$ representation) corresponding to $\lambda, E_{\lambda} \in Z\left(\mathbb{C}\left[S_{n}\right]\right)$ its associated Young idempotent (or central idempotent), and $\chi^{\lambda}: \mathbb{C}\left[S_{n}\right] \rightarrow \mathbb{C}$ its associated character. Abusing notation, we write $\chi^{\lambda}(\mu)$ for $\chi^{\lambda}(g)$ when $\operatorname{sh}(g)=\mu$ (this notation is well-defined since $\chi^{\lambda}$ is a class function). The normalized character $\tilde{\chi}^{\lambda}: \bigoplus_{k \leqslant n} \mathbb{C}\left[S_{k}\right] \rightarrow \mathbb{C}$ associated to $\lambda$ is defined so that for $x \in \mathbb{C}\left[S_{k}\right]$,

$$
\tilde{\chi}^{\lambda}(x):=\frac{\chi^{\lambda}\left(\iota_{k, n}(x)\right)}{\operatorname{dim} L^{\lambda}}=\frac{\chi^{\lambda}\left(\iota_{k, n}(x)\right)}{\chi^{\lambda}\left(1_{n}\right)} .
$$

Let $\mu=\left(\mu_{1}, \ldots, \mu_{t}\right) \vdash k \leqslant n$ and set $\pi_{\mu}=1_{k}$ if $\mu=\left(1^{k}\right)$ and otherwise

$$
\begin{aligned}
\pi_{\mu} & =\left(s_{k-1} \ldots s_{k-\mu_{t}+1}\right) \ldots\left(s_{\mu_{1}+\mu_{2}-1} \ldots s_{\mu_{1}+1}\right) \cdot\left(s_{\mu_{1}-1} \ldots s_{2} s_{1}\right) \\
& =\left(k, k-1, \ldots, k-\mu_{t}+1\right) \ldots\left(\mu_{1}+\mu_{2}, \ldots, \mu_{1}+1\right) \cdot\left(\mu_{1}, \ldots, 2,1\right) \in S_{k} .
\end{aligned}
$$

We define

$$
\sigma_{\mu, n}:=w_{0, n}^{-1}\left(\iota_{k, n}\left(\pi_{\mu}\right)\right) w_{0, n} \in S_{n} .
$$

Observe that $\sigma_{\mu, n}$ has cycle type $\phi_{k, n}(\mu)$ and fixes $1,2, \ldots, n-k$ pointwise. 
ExAmPLE 2.5. Let $\mu=(3,2) \vdash 5$, then

$$
\pi_{\mu}=\left(s_{4}\right)\left(s_{2} s_{1}\right)=(5,4)(3,2,1)
$$

and we see that $\operatorname{sh}\left(\pi_{\mu}\right)=\mu$. For $n=8$,

$$
\sigma_{\mu, 8}=s_{4} s_{6} s_{7}=(4,5)(6,7,8),
$$

while for $n=10$,

$$
\sigma_{\mu, 10}=(6,7)(8,9,10)
$$

The elements

$$
\left\{\sigma_{(1), n}, \sigma_{(2), n}, \sigma_{(3), n}, \ldots, \sigma_{(n), n}\right\}=\left\{1_{n}, s_{n-1}, s_{n-2} s_{n-1}, \ldots, s_{1} s_{2} \ldots s_{n-1}\right\}
$$

are the minimal length left coset representatives of $S_{n-1}$ in $S_{n}$. We extend this observation in the following lemma.

LEMMA 2.6. For $k<n$, the elements of the set

$$
\left\{\sigma_{\left(i_{n}\right), n} \sigma_{\left(i_{n-1}\right), n-1} \ldots \sigma_{\left(i_{k+1}\right), k+1} \mid 1 \leqslant i_{j} \leqslant j\right\}
$$

are the minimal length left coset representatives of $S_{k}$ in $S_{n}$. We denote this set by $\mathcal{L C}_{k}^{n}$.

Proof. Let $G_{n-2} \subset G_{n-1} \subset G_{n}$ be a sequence of nested groups and $\left\{g_{1}^{(n-1)}, \ldots, g_{t_{n-1}}^{(n-1)}\right\}$ and $\left\{g_{1}^{(n)}, \ldots, g_{t_{n}}^{(n)}\right\}$ be a collection of left coset representatives of $G_{n-2}$ in $G_{n-1}$ and $G_{n-1}$ in $G_{n}$ respectively. Then $\left\{g_{i_{2}}^{(n)} g_{i_{1}}^{(n-1)} \mid 1 \leqslant i_{1} \leqslant t_{n-1}\right.$ and $\left.1 \leqslant i_{2} \leqslant t_{n}\right\}$ is a collection of left coset representatives of $G_{n-2}$ in $G_{n}$. This fact can be extended inductively to calculate the left coset representatives of $G_{k}$ in $G_{n}$ for any nested sequence $G_{k} \subset G_{k+1} \subset \cdots \subset G_{n}$ when a collection of left coset representatives of $G_{i-1}$ in $G_{i}$ is known for $k+1 \leqslant i \leqslant n$. The lemma then follows from the observation that

$$
\begin{aligned}
\left\{\sigma_{\left(i_{j}\right), j} \mid 1 \leqslant i_{j} \leqslant j\right\} & =\left\{\sigma_{(1), j}, \sigma_{(2), j}, \ldots, \sigma_{(j), j}\right\} \\
& =\left\{1_{j}, s_{j-1}, s_{j-2} s_{j-1}, \ldots, s_{1} s_{2} \ldots s_{j-1}\right\}
\end{aligned}
$$

is a collection of left coset representatives of $S_{j-1}$ in $S_{j}$.

We note that $\left|\mathcal{L C}_{k}^{n}\right|=(n\lfloor n-k)$, where the falling factorial power is defined as

$$
(x \downarrow j)= \begin{cases}x(x-1) \ldots(x-j+1), & \text { if } j=1,2, \ldots \\ 1, & \text { if } j=0 .\end{cases}
$$

EXAMPLE 2.7. We have

$$
\begin{gathered}
\mathcal{L C}_{3}^{4}=\left\{1_{4}, s_{3}, s_{2} s_{3}, s_{1} s_{2} s_{3}\right\} \\
\mathcal{L C}_{2}^{3}=\left\{1_{3}, s_{2}, s_{1} s_{2}\right\}
\end{gathered}
$$

and

$$
\begin{aligned}
\mathcal{L C}_{2}^{4}=\{ & 1_{4}, s_{3}, s_{2} s_{3}, s_{1} s_{2} s_{3}, \\
& s_{2}, s_{3} s_{2}, s_{2} s_{3} s_{2}, s_{1} s_{2} s_{3} s_{2}, \\
& \left.s_{1} s_{2}, s_{3} s_{1} s_{2}, s_{2} s_{3} s_{1} s_{2}, s_{1} s_{2} s_{3} s_{1} s_{2}\right\}
\end{aligned}
$$


2.1. The CENTER OF $\mathbb{C}\left[S_{n}\right]$. For $\mu \vdash k \leqslant n$, set

$$
C_{\mu, n}:=\sum_{\substack{g \in S_{n}, \operatorname{sh}(g)=\phi_{k, n}(\mu)}} g .
$$

The elements $\left\{C_{\mu, n}\right\}_{\mu \vdash n}$ are a basis for the center of the symmetric group algebra, $Z\left(\mathbb{C}\left[S_{n}\right]\right)$. We write $z_{\mu, n}$ for the size of the centralizer of an element in $S_{n}$ with cycle type $\phi_{k, n}(\mu)$. Note that when $\mu \vdash n$, then $z_{\mu, n}=z_{\mu}$.

DEFinition 2.8. For $\mu=\left(\mu_{1}, \ldots, \mu_{t}\right) \vdash k \leqslant n$, set

$$
A_{\mu, n}:=\sum_{g \in \mathcal{L C}_{n-k}^{n}} g \sigma_{\mu, n} g^{-1} .
$$

$A_{\mu, n}$ is called the normalized conjugacy class sum associated to $\mu$ in $\mathbb{C}\left[S_{n}\right]$.

Alternatively, $A_{\mu, n}$ may be written as

$$
A_{\mu, n}=\sum\left(i_{1}, \ldots, i_{\mu_{1}}\right) \ldots\left(i_{k-\mu_{t}+1}, \ldots, i_{k}\right)
$$

where this sum is taken over all distinct $k$-tuples $\left(i_{1}, \ldots, i_{k}\right)$ of elements from $\{1,2, \ldots, n\}$. From (5) a counting argument shows that

$$
A_{\mu, n}=\frac{z_{\mu, n}}{(n-k) !} C_{\mu, n}
$$

It follows from (6) that $A_{\mu, n} \in Z\left(\mathbb{C}\left[S_{n}\right]\right)$.

ExAMPLE 2.9. Let $k \leqslant n$. When $\mu=(k) \vdash k$, then $z_{(k), n}=k(n-k)$ ! so that

$$
A_{(k), n}=k C_{(k), n} .
$$

The elements $A_{\mu, n}$ are important in the study of the asymptotic character theory of symmetric groups [12]. They also appear in connection with the algebra of partial permutations [8]. If $\mu \vdash k \leqslant n$ and $\lambda \vdash n$ then

$$
\tilde{\chi}^{\lambda}\left(A_{\mu, n}\right)=(n \downarrow k) \frac{\chi^{\lambda}\left(\phi_{k, n}(\mu)\right)}{\operatorname{dim} L^{\lambda}} .
$$

The following is well-known.

Proposition 2.10. When restricted to $Z\left(\mathbb{C}\left[S_{n}\right]\right)$, the normalized character $\tilde{\chi}^{\lambda}$ is an algebra homomorphism from $Z\left(\mathbb{C}\left[S_{n}\right]\right)$ to $\mathbb{C}$.

$Z\left(\mathbb{C}\left[S_{n}\right]\right)$ is also generated by symmetric polynomials in the Jucys-Murphy elements $\left\{J_{i}\right\}_{1 \leqslant i \leqslant n} \subseteq \mathbb{C}\left[S_{n}\right]$, where

$$
J_{1}=0, \quad \text { and } \quad J_{k}=(1, k)+(2, k)+\cdots+(k-1, k), \quad 2 \leqslant k \leqslant n .
$$

Written in terms of the Coxeter generators

$$
J_{k}=\sum_{i=1}^{k-1} s_{i} \ldots s_{k-2} s_{k-1} s_{k-2} \ldots s_{i} .
$$

2.2. The transition measure and CO-Transition Measure. In this section we recall the notion of transition and co-transition measures, also known as growth and decay, respectively. Assume that $\lambda \vdash n$ and let $a_{1}, \ldots, a_{d}$ and $b_{1}, \ldots, b_{d-1}$ be the interlacing sequences associated to $\lambda$. Recall that $\lambda^{(1)}, \ldots, \lambda^{(d)}$ are the partitions of $n+1$ such that $\operatorname{cont}\left(\lambda^{(i)} / \lambda\right)=a_{i}$, while $\lambda_{(1)}, \ldots, \lambda_{(d-1)}$ are the partitions of $n-1$ such that $\operatorname{cont}\left(\lambda / \lambda_{(i)}\right)=b_{i}$. 
For $1 \leqslant i \leqslant d$, the transition probabilities for $\lambda$ are defined as

$$
\widehat{q}_{\lambda}\left(\lambda^{(i)}\right):=\frac{\operatorname{dim}\left(L^{\lambda^{(i)}}\right)}{(n+1) \operatorname{dim}\left(L^{\lambda}\right)} .
$$

The transition measure $\widehat{\omega}_{\lambda}$ is then the probability measure on $\mathbb{R}$ defined by

$$
\widehat{\omega}_{\lambda}:=\sum_{i=1}^{d} \widehat{q}_{\lambda}\left(\lambda^{(i)}\right) \delta_{a_{i}}
$$

where $\delta_{a_{i}}$ is the Dirac delta measure with support on $a_{i} \in \mathbb{R}$. Dually, for $1 \leqslant i \leqslant d-1$ the co-transition probabilities of $\lambda$ are

$$
\breve{q}_{\lambda}\left(\lambda_{(i)}\right):=\frac{\operatorname{dim}\left(L^{\lambda_{(i)}}\right)}{\operatorname{dim}\left(L^{\lambda}\right)}
$$

and the co-transition measure $\breve{\omega}_{\lambda}$ is

$$
\breve{\omega}_{\lambda}:=\sum_{i=1}^{d-1} \breve{q}_{\lambda}\left(\lambda_{(i)}\right) \delta_{b_{i}} .
$$

These probability measures were first investigated by Kerov ([10, 11]). They are fundamental tools in the study of the asymptotic representation theory of symmetric groups. For example, in [11] Kerov shows that

$$
\sum_{i=1}^{d} \frac{\widehat{q}_{\lambda}\left(\lambda^{(i)}\right)}{z-a_{i}}=\frac{\left(z-b_{1}\right) \ldots\left(z-b_{d-1}\right)}{\left(z-a_{1}\right) \ldots\left(z-a_{d-1}\right)\left(z-a_{d}\right)}
$$

and

$$
z-|\lambda| \sum_{i=1}^{d-1} \frac{\check{q}_{\lambda}\left(\lambda_{(i)}\right)}{z-b_{i}}=\frac{\left(z-a_{1}\right) \ldots\left(z-a_{d-1}\right)\left(z-a_{d}\right)}{\left(z-b_{1}\right) \ldots\left(z-b_{d-1}\right)} .
$$

The $k$ th moment associated to the transition measure $\widehat{\omega}_{\lambda}$ is given by

$$
\widehat{m}_{k}(\lambda)=\sum_{i=1}^{d} a_{i}^{k} \widehat{q}_{\lambda}\left(\lambda^{(i)}\right)
$$

while the $k$ th moment associated to the co-transition measure $\breve{\omega}_{\lambda}$ is given by

$$
\check{m}_{k}(\lambda)=\sum_{i=1}^{d-1} b_{i}^{k} \check{q}_{\lambda}\left(\lambda_{(i)}\right)
$$

Consider the series

$$
\widehat{\mathcal{M}}_{\lambda}(z):=\sum_{k=0}^{\infty} \widehat{m}_{k}(\lambda) z^{-k-1} \quad \text { and } \quad \widetilde{\mathcal{M}}_{\lambda}(z):=z-\sum_{k=0}^{\infty}|\lambda| \breve{m}_{k}(\lambda) z^{-k-1} .
$$

LEMma 2.11. For $\lambda \in \mathcal{P}$

$$
\widehat{\mathcal{M}}_{\lambda}(z)=\left(\widetilde{\mathcal{M}}_{\lambda}(z)\right)^{-1}
$$

Proof. This follows directly from equations (11), (12) and Lemma 5.1 in [11].

Boolean cumulants linearize convolution of probability measures under the notion of Boolean independence [19]. The most convenient way to define the boolean cumulants $\left\{\hat{b}_{k}(\lambda)\right\}_{k \geqslant 1}$ associated to $\hat{\omega}_{\lambda}$ in our case is as the coefficients on the multiplicative inverse of $\widehat{\mathcal{M}}_{\lambda}(z)$. We write

$$
\widehat{\mathcal{B}}_{\lambda}(z)=z-\sum_{k=-1}^{\infty} \widehat{b}_{k+2}(\lambda) z^{-k-1}=\left(\widehat{\mathcal{M}}_{\lambda}(z)\right)^{-1}
$$


(for the equivalence of this definition and other analytic definitions see Section 2 of [19]). Given Lemma 2.11 this definition immediately implies the following proposition.

Proposition 2.12. Let $\lambda \in \mathcal{P}$ and $k \geqslant 0$, then $\widehat{b}_{1}(\lambda)=0$ and

$$
\widehat{b}_{k+2}(\lambda)=|\lambda| \check{m}_{k}(\lambda) \text {. }
$$

REMARK 2.13. The equality (14) can be rewritten as

$$
\sum_{i=1}^{k} \widehat{m}_{k-i}(\lambda) \hat{b}_{i}(\lambda)=\widehat{m}_{k}(\lambda)
$$

For general information about the relationship between moments, Boolean cumulants, and other families of cumulants see [1].

There is a more algebraic approach to the transition measure due to Biane [2]. Let

$$
\operatorname{pr}_{n-1}: \mathbb{C}\left[S_{n}\right] \rightarrow \mathbb{C}\left[S_{n-1}\right] \subset \mathbb{C}\left[S_{n}\right]
$$

be the projection map defined on $S_{n}$ by

$$
\operatorname{pr}_{n-1}(g)= \begin{cases}g & \text { if } g \in \mathbb{C}\left[S_{n-1}\right] \\ 0 & \text { otherwise }\end{cases}
$$

In the context of probability theory, $\mathrm{pr}_{n-1}$ is sometimes known as the conditional expectation.

Proposition 2.14. For $\lambda \vdash n$,

$$
\widehat{m}_{k}(\lambda)=\widetilde{\chi}^{\lambda}\left[\operatorname{pr}_{n}\left(J_{n+1}^{k}\right)\right]
$$

and

$$
\widehat{b}_{k+2}(\lambda)=|\lambda| \breve{m}_{k}(\lambda)=\widetilde{\chi}^{\lambda}\left(\sum_{i=1}^{n} s_{i} \ldots s_{n-1} J_{n}^{k} s_{n-1} \ldots s_{i}\right) .
$$

Proof. The statement of (17) appears in [3, Section 4]. A detailed proof is given in Theorem 9.23 of [7]. To get (18) note that since characters are class functions,

$$
\tilde{\chi}^{\lambda}\left(\sum_{i}^{n} s_{i} \ldots s_{n-1} J_{n}^{k} s_{n-1} \ldots s_{i}\right)=|\lambda| \widetilde{\chi}^{\lambda}\left(J_{n}^{k}\right) .
$$

As $J_{n}$ eigenspaces, $L^{\lambda}$ decomposes as

$$
L^{\lambda} \cong \bigoplus_{i=1}^{d-1} L^{\lambda_{(i)}}
$$

with $L^{\lambda_{(i)}}$ corresponding to eigenvalue $b_{i}[21]$. Hence,

$$
|\lambda| \tilde{\chi}^{\lambda}\left(J_{n}^{k}\right)=|\lambda| \sum_{i=1}^{d-1} \frac{\operatorname{dim}\left(\lambda_{(i)}\right) b_{i}^{k}}{\operatorname{dim}(\lambda)}=|\lambda| \check{m}_{k}(\lambda)=\widehat{b}_{k+2}(\lambda) .
$$

Proposition 2.14 is related to the fact that we are working in a noncommutative probability space (that is, a von Neumann algebra equipped with a normal faithful trace). In our case the algebra is $\operatorname{End}\left(L^{\lambda}\right) \otimes M_{n+1}(\mathbb{C})$ and $\widehat{\omega}_{\lambda}$ then arises from the distribution of a self-adjoint element in this algebra (see Proposition 3.3 in [2]). 


\section{Symmetric FUnCtions And SHIfTED SyMmetric FUnCtions}

In order to define the algebra of shifted symmetric functions, we first recall the classical symmetric functions. Let $\Lambda_{n}$ be the algebra of symmetric polynomials over $\mathbb{C}$ in $x_{1}, \ldots, x_{n}$. This algebra is graded by polynomial degree. Recall that for $n \geqslant 0$ there is a homomorphism

$$
\Lambda_{n+1} \rightarrow \Lambda_{n}
$$

given by setting $x_{n+1}=0$ in $\Lambda_{n+1}$. One can define the algebra of symmetric functions as the projective limit $\Lambda=\lim \Lambda_{n}$ taken in the category of graded algebras. We recall three collections of algebraically independent generators of $\Lambda$ :

- elementary symmetric functions $e_{1}, e_{2}, e_{3}, \ldots$,

- complete homogeneous symmetric functions $h_{1}, h_{2}, h_{3}, \ldots$,

- power sum symmetric functions $p_{1}, p_{2}, p_{3}, \ldots$

For $\left\{f_{k}\right\}_{k \geqslant 1}$ equal to any of these three sets of generators and $\lambda=\left(\lambda_{1}, \ldots, \lambda_{r}\right)$ we write $f_{\lambda}:=f_{\lambda_{1}} \ldots f_{\lambda_{r}}$. We denote the basis of Schur functions by $\left\{s_{\lambda}\right\}_{\lambda \in \mathcal{P}}$. We refer the reader to [16] and [20] for background on $\Lambda$.

Let $\Lambda_{n}^{*}$ be the algebra of polynomials over $\mathbb{C}$ in $x_{1}, \ldots, x_{n}$, which become symmetric in the new variables $x_{i}^{\prime}=x_{i}-i$. This algebra is filtered by polynomial degree. In analogy to $\Lambda_{n+1}$, setting $x_{n+1}=0$ in $\Lambda_{n+1}^{*}$ gives a homomorphism

$$
\Lambda_{n+1}^{*} \rightarrow \Lambda_{n}^{*}
$$

which respects the filtration. Using (20), set

$$
\Lambda^{*}:=\lim _{\longleftarrow} \Lambda_{n}^{*}
$$

where this limit is taken in the category of filtered algebras. $\Lambda^{*}$ is called the algebra of shifted symmetric functions.

Because $\Lambda^{*}$ is filtered, we can consider the associated graded algebra $\operatorname{gr}\left(\Lambda^{*}\right)$.

Proposition $3.1([18$, Proposition 1.5$]) \cdot \operatorname{gr}\left(\Lambda^{*}\right)$ is canonically isomorphic to $\Lambda$.

REMARK 3.2. It is noted in Remark 1.7 of [18] that we may also view $\Lambda^{*}$ as a deformation of $\Lambda$. Let $\Lambda_{n}^{*}(\theta)$ be the algebra of polynomials in $x_{1}, \ldots, x_{n}$ which are symmetric in the new variables $x_{i}^{\prime}=x_{i}+c-i \theta$ for $1 \leqslant i \leqslant n$ and where $c \in \mathbb{C}$. Define $\Lambda^{*}(\theta)=\lim _{\longleftarrow} \Lambda_{n}^{*}(\theta)$. Then $\Lambda^{*}(0)=\Lambda$ and $\Lambda^{*}(1)=\Lambda^{*}$. In fact for all $\theta \neq 0, \Lambda^{*}(\theta) \cong \Lambda^{*}$.

3.1. BASES OF $\Lambda^{*}$. In [18] Okounkov and Olshanski introduced a remarkable basis for $\Lambda^{*}$ called the shifted Schur functions. Let $\lambda=\left(\lambda_{1}, \ldots, \lambda_{n}\right)$ be a partition with $\lambda_{1} \geqslant \cdots \geqslant \lambda_{n} \geqslant 0$ (note that here we allow components of a partition to be zero). The shifted Schur polynomial in $n$ variables, indexed by $\lambda$ is the ratio of two $n \times n$ determinants,

$$
s_{\lambda}^{*}\left(x_{1}, \ldots, x_{n}\right)=\frac{\operatorname{det}\left[\left(x_{i}+n-i\left\lfloor\lambda_{j}+n-j\right)\right]\right.}{\operatorname{det}\left[\left(x_{i}+n-i\lfloor n-j)\right]\right.},
$$

where $1 \leqslant i, j \leqslant n$ and $\left(x\lfloor k)\right.$ is defined in (3). This polynomial belongs to $\Lambda_{n}^{*}$. It is shown in [18] that

$$
s_{\lambda}^{*}\left(x_{1}, \ldots, x_{n}, 0\right)=s_{\lambda}^{*}\left(x_{1}, \ldots, x_{n}\right) .
$$

This implies that for fixed $\lambda$, letting $n \rightarrow \infty$ gives a well-defined element $s_{\lambda}^{*}$ of $\Lambda^{*}$. The elements $\left\{s_{\lambda}^{*}\right\}_{\lambda \in \mathcal{P}} \in \Lambda^{*}$ are called the shifted Schur functions and form a basis for $\Lambda^{*}$. There is a linear map $\Lambda^{*} \rightarrow \operatorname{gr}\left(\Lambda^{*}\right) \cong \Lambda$ which sends $f \in \Lambda^{*}$ to its top homogeneous component which is an element of $\Lambda$. Under this map

$$
s_{\lambda}^{*} \mapsto s_{\lambda}
$$


or alternatively,

$$
s_{\lambda}^{*}=s_{\lambda}+\text { l.o.t. }
$$

where l.o.t. means lower order terms in polynomial degree.

In analogy to the classical case, the elementary shifted functions can be defined as $e_{k}^{*}:=s_{\left(1^{k}\right)}^{*}$, while the complete shifted functions can be defined as $h_{k}^{*}:=s_{(k)}^{*}$. More explicitly:

$$
e_{k}^{*}\left(x_{1}, x_{2}, \ldots\right)=\sum_{1 \leqslant i_{1}<\cdots<i_{k}<\infty}\left(x_{i_{1}}+k-1\right)\left(x_{i_{2}}+k-2\right) \ldots x_{i_{k}}
$$

and

$$
h_{k}^{*}\left(x_{1}, x_{2}, \ldots\right)=\sum_{1 \leqslant i_{1} \leqslant \cdots \leqslant i_{k}<\infty}\left(x_{i_{1}}-k+1\right)\left(x_{i_{2}}-k+2\right) \ldots x_{i_{k}} .
$$

Let $F$ be the linear isomorphism $F: \Lambda \rightarrow \Lambda^{*}$ which sends $s_{\lambda} \mapsto s_{\lambda}^{*}$. Define the element $p_{\lambda}^{\#} \in \Lambda^{*}$ to then be

$$
p_{\lambda}^{\#}:=F\left(p_{\lambda}\right)
$$

where $p_{\lambda}$ is the power sum symmetric function. The elements $p_{\lambda}^{\#}$ are one of several shifted analogues of the power sums. For $\lambda \vdash n$, the transition coefficients between the power-sum and Schur bases are given by the character tables of the symmetric group (see [20]):

$$
p_{\lambda}=\sum_{\mu \vdash n} \chi^{\mu}(\lambda) s_{\mu}
$$

It follows directly from definition (24) that

$$
p_{\lambda}^{\#}=\sum_{\mu \vdash n} \chi^{\mu}(\lambda) s_{\mu}^{*}
$$

Note also that by (23) and (25),

$$
p_{\lambda}^{\#}=p_{\lambda}+\text { l.o.t. }
$$

Since the power symmetric functions $p_{1}, p_{2}, \ldots$ are algebraically independent and generate $\Lambda$, it follows from Proposition 3.1 and (26) that $p_{1}^{\#}, p_{2}^{\#}, \ldots$ are algebraically independent and generate $\Lambda^{*}$. Similarly, since $\left\{p_{\lambda}\right\}_{\lambda \in \mathcal{P}}$ is a basis for $\Lambda,\left\{p_{\lambda}^{\#}\right\}_{\lambda \in \mathcal{P}}$ is a basis for $\Lambda^{*}$. For more properties of the basis $\left\{p_{\lambda}^{\#}\right\}$ see [9].

REMARK 3.3. Let $\lambda=\left(\lambda_{1}, \ldots, \lambda_{r}\right) \vdash n$. While it is true that in $\Lambda, p_{\lambda_{1}} \ldots p_{\lambda_{r}}=p_{\lambda}$, in general

However, by (26)

$$
p_{\lambda_{1}}^{\#} \ldots p_{\lambda_{r}}^{\#} \neq p_{\lambda}^{\#}
$$

$$
p_{\lambda_{1}}^{\#} \ldots p_{\lambda_{r}}^{\#}=p_{\lambda}^{\#}+\text { l.o.t. }
$$

3.2. $\Lambda^{*}$ AS FUnCtions on $\mathcal{P}$. Let $\operatorname{Fun}(\mathcal{P}, \mathbb{C})$ be the algebra of functions from $\mathcal{P}$ to $\mathbb{C}$ with pointwise multiplication. Viewing $\mu=\left(\mu_{1}, \ldots, \mu_{t}\right) \vdash k$ as the sequence $\left(\mu_{1}, \ldots, \mu_{t}, 0,0, \ldots\right)$, we can evaluate $f \in \Lambda^{*}$ on $\mu$ by setting

$$
f(\mu)=f\left(\mu_{1}, \ldots, \mu_{t}, 0,0, \ldots\right) .
$$

Since $\left(\mu_{1}, \ldots, \mu_{t}, 0,0, \ldots\right)$ has only a finite number of nonzero values, it is clear that $(27)$ is well-defined. In fact $f$ is uniquely defined by its values on $\mathcal{P}$. Thus $\Lambda^{*}$ may be realized as a subalgebra of $\operatorname{Fun}(\mathcal{P}, \mathbb{C})$. This fact is used repeatedly en route to establishing many of the fundamental results about shifted symmetric functions in [12] and [18]. 
For $\lambda=\left(\lambda_{1}, \ldots, \lambda_{r}\right) \vdash n$ and $\alpha$ a cell in the Young diagram corresponding to $\lambda$ with coordinates $(i, j)$, the hook length of $\alpha$ is defined as $h(\alpha):=\lambda_{i}-j+\lambda_{j}^{\prime}-i+1$, where $\lambda^{\prime}=\left(\lambda_{1}^{\prime}, \ldots, \lambda_{\lambda_{1}}^{\prime}\right)$ is the partition conjugate to $\lambda$. Set $H(\lambda)$ as the product of all hooklengths in $\lambda$,

$$
H(\lambda):=\prod_{\alpha \in \lambda} h(\alpha)
$$

The following is known as the "Characterization Theorem" of [17].

THEOREM 3.4. For $\mu \vdash k, s_{\mu}^{*}$ is the unique element of $\Lambda^{*}$ such that $\operatorname{deg}\left(s_{\mu}^{*}\right) \leqslant k$ and

$$
s_{\mu}^{*}(\lambda)=\delta_{\mu \lambda} H(\mu)
$$

for all $\lambda \in \mathcal{P}$ such that $|\lambda| \leqslant|\mu|$.

This theorem along with (25) then give the following proposition.

Proposition 3.5 ([18]). For $\mu \vdash k, \lambda \vdash n$,

$$
p_{\mu}^{\#}(\lambda)= \begin{cases}\frac{(n \mid k)}{\operatorname{dim} L^{\lambda}} \chi^{\lambda}\left(\phi_{k, n}(\mu)\right) & k \leqslant n \\ 0 & \text { otherwise. }\end{cases}
$$

REMARK 3.6. We will later use the fact that $p_{1}^{\#}=x_{1}+x_{2}+\cdots=p_{1}$, so that $p_{1}^{\#}(\lambda)=|\lambda|$ for all $\lambda \in \mathcal{P}$.

In Section 2.2 we introduced the moments $\left\{\widehat{m}_{k}(\lambda)\right\}$ (resp. $\left\{\breve{m}_{k}(\lambda)\right\}$ ) of the transition measure (resp. co-transition measure) associated to a partition $\lambda$ and the corresponding Boolean cumulants $\left\{\hat{b}_{k}(\lambda)\right\}$. We can interpret all of these as elements of $\operatorname{Fun}(\mathcal{P}, \mathbb{C})$ via

$$
\lambda \stackrel{\widehat{m}_{k}}{\longmapsto} \widehat{m}_{k}(\lambda), \quad \lambda \stackrel{\breve{m}_{k}}{\longmapsto} \check{m}_{k}(\lambda), \quad \text { and } \quad \lambda \stackrel{\hat{b}_{k}}{\longmapsto} \widehat{b}_{k}(\lambda) .
$$

Henceforth we omit the partition argument from $\widehat{m}_{k}, \check{m}_{k}$, and $\hat{b}_{k}$ in cases where we want to emphasize that we are considering these as elements of $\operatorname{Fun}(\mathcal{P}, \mathbb{C})$.

Proposition 3.7 ([15, Theorem 6.4]). As elements of $\operatorname{Fun}(\mathcal{P}, \mathbb{C}), \hat{m}_{k}$ and $\hat{b}_{k}$ belong to $\Lambda^{*}$.

REMARK 3.8. In [15] Section 5, Lassalle shows that with the appropriate alphabet $A_{\lambda}$ (which is specific to each partition $\lambda$ ),

$$
\widehat{m}_{k}(\lambda)=h_{k}\left(A_{\lambda}\right) \quad \text { and } \quad \hat{b}_{k}(\lambda)=(-1)^{k-1} e_{k}\left(A_{\lambda}\right)
$$

\section{The Heisenberg Category $\mathcal{H}^{\prime}$}

In [13], Khovanov defines an additive $\mathbb{C}$-linear monoidal category $\mathcal{H}^{\prime}$ which we will call the Heisenberg category. The objects in $\mathcal{H}^{\prime}$ are generated by two objects $Q_{+}$and $Q_{-}$. Following the notation of [13], we denote $Q_{\epsilon_{1}} \otimes \cdots \otimes Q_{\epsilon_{m}}$ by $Q_{\epsilon}$ where $\epsilon=\epsilon_{1} \ldots \epsilon_{m}$ is a finite sequence of pluses and minuses. The unit object, $\mathbb{1}$, corresponds to the empty sequence $Q_{\varnothing}$.

The collection of morphisms $\operatorname{Hom}_{\mathcal{H}^{\prime}}\left(Q_{\epsilon}, Q_{\epsilon^{\prime}}\right)$, for two sequences $\epsilon$ and $\epsilon^{\prime}$ is the $\mathbb{C}$ vector space spanned by planar diagrams modulo some local relations. The diagrams are oriented compact 1 -manifolds immersed in the strip $\mathbb{R} \times[0,1]$, modulo rel boundary isotopies. The endpoints of the 1 -manifolds are located at $\{1, \ldots, m\} \times\{0\}$ and $\{1, \ldots, n\} \times\{1\}$, where $m$ and $n$ are the lengths of $\epsilon$ and $\epsilon^{\prime}$, respectively. Further, the orientation of the 1-manifolds at the endpoints must match the signs in the sequences $\epsilon$ and $\epsilon^{\prime}$. Triple intersections are not allowed. 
EXAMPLE 4.1. The diagram

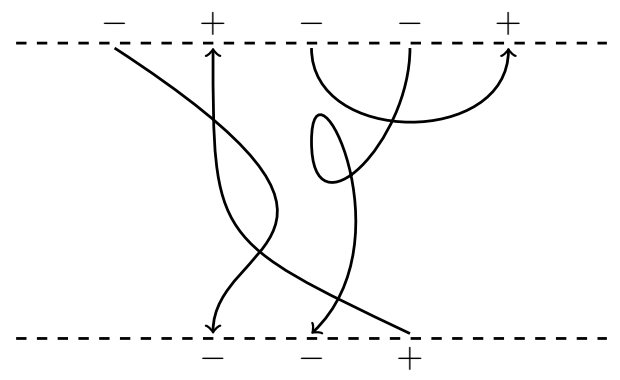

is a morphism from $Q_{--+}$to $Q_{-+--+}$.

The composition of two morphisms is achieved by stacking diagrams. The local relations for diagrams are:
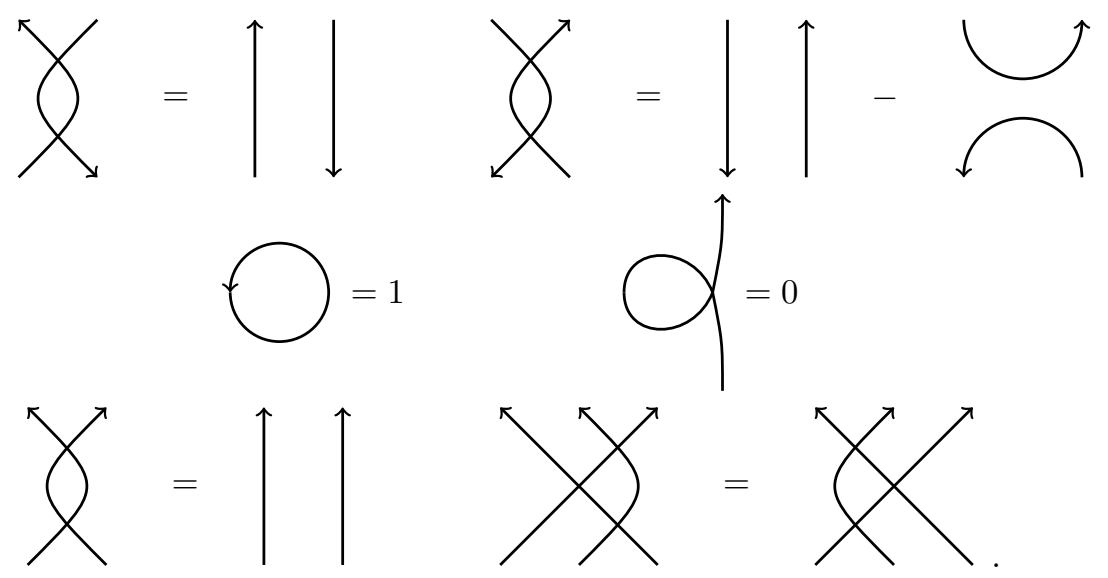

The relations (30) and (31) are motivated by the Heisenberg relation $p q=q p+1$, while the relations (32) are motivated by the symmetric group relations.

It is convenient to denote a right curl by a dot on a strand, and a sequence of $d$ right curls by a dot with a $d$ next to it:

$$
\{:=d \uparrow:=\{\} d \operatorname{dots}
$$

Using relations (30)-(32) it can be shown that a dot can be moved across intersection points, according to the following "dot-sliding relations" [13]:

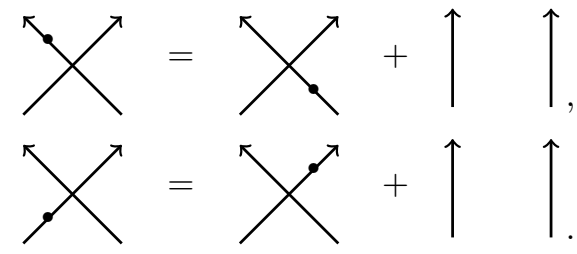

This observation easily generalizes to

$$
\nwarrow k \nearrow=\searrow_{k}^{k-1}+\sum_{i=0}^{k \uparrow} i k-1-i
$$




$$
\nwarrow \searrow=\searrow^{k}=\sum_{i=0}^{k-1} i \uparrow \uparrow k-1-i
$$

Another consequence of relations (30)-(32) are the "bubble moves" [13]:

$$
k \bigcirc \uparrow \uparrow \uparrow+(k+1) \uparrow k-\sum_{i=0}^{k-2}(k-i-1) \uparrow k-i-2 \quad i \bigcirc
$$

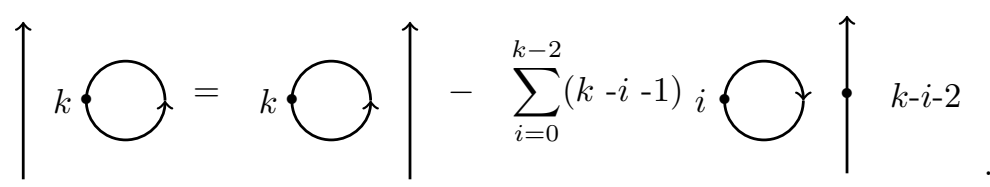

Note that relations (32) imply that there is a homomorphism $\mathcal{T}_{n}: \mathbb{C}\left[S_{n}\right] \rightarrow$ $\operatorname{End}_{\mathcal{H}^{\prime}}\left(Q_{+^{n}}\right)$ which sends

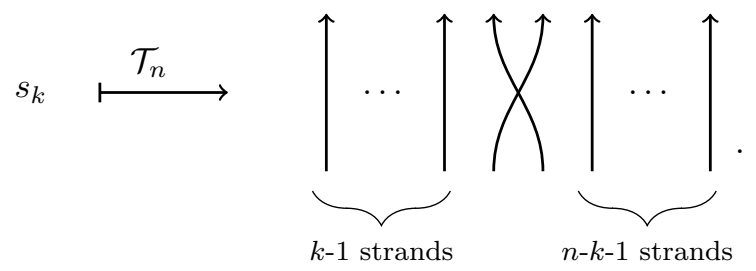

Diagrammatically, for $x \in \mathbb{C}\left[S_{n}\right]$ we set

$$
\mathcal{T}_{n}(x)=: \underbrace{\uparrow \uparrow \cdots \uparrow}_{n \text { strands }} .
$$

The appearance of the group algebra $\mathbb{C}\left[S_{n}\right]$ as endomorphisms in $\mathcal{H}^{\prime}$ is responsible for the connection between $\mathcal{H}^{\prime}$ and the representation theory of symmetric groups.

4.1. The endomorphism algebra $\operatorname{End}_{\mathcal{H}^{\prime}}(\mathbb{1})$. The center of $\mathcal{H}^{\prime}$ is $\operatorname{End}_{\mathcal{H}^{\prime}}(\mathbb{1})$, that is, the algebra of endomorphisms of the monoidal unit object $\mathbb{1}$. Diagrammatically, the algebra $\operatorname{End}_{\mathcal{H}^{\prime}}(\mathbb{1})$ is the commutative $\mathbb{C}$-algebra spanned by all closed diagrams, with multiplication given by juxtaposition of diagrams. The algebra structure of $\operatorname{End}_{\mathcal{H}^{\prime}}(\mathbb{1})$ was determined by Khovanov in [13]. Let $\mathbb{C}\left[c_{0}, c_{1}, c_{2}, \ldots\right]$ be the polynomial algebra in countably many indeterminants $\left\{c_{i}\right\}_{i \geqslant 0}$.

Theorem 4.2 ([13, Proposition 3]). The map $\psi_{0}: \mathbb{C}\left[c_{0}, c_{1}, \ldots\right] \rightarrow \operatorname{End}_{\mathcal{H}^{\prime}}(\mathbb{1})$ which sends

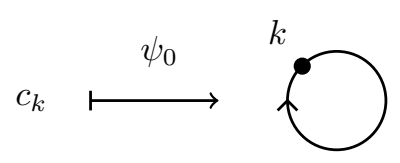

is an algebra isomorphism.

Henceforth we will freely identify $c_{k}$ with its image in $\operatorname{End}_{\mathcal{H}^{\prime}}(\mathbb{1})$. Another natural set of diagrams to consider are the counterclockwise-oriented circles with $k$ right-twist curls on them. Set 


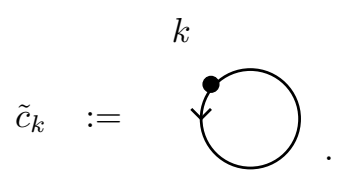

It follows from the relations in (31) that $\tilde{c}_{0}=1$ and $\tilde{c}_{1}=0$.

Lemma 4.3 ([13, Proposition 2]). For $k>0$,

$$
\tilde{c}_{k+1}=\sum_{i=0}^{k-1} c_{k-1-i} \tilde{c}_{i} .
$$

Another class of elements in $\operatorname{End}_{\mathcal{H}^{\prime}}(\mathbb{1})$ we consider are those arising from the closure of permutations (that is, closures of morphisms in the image of $\mathcal{T}_{n}$ ). We define

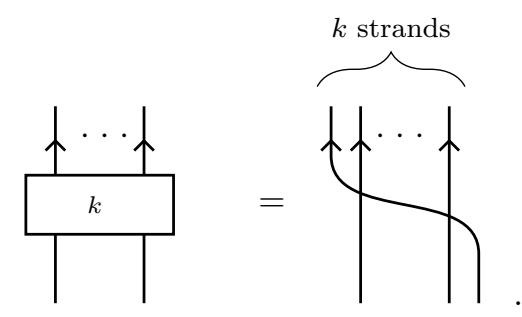

For $\lambda=\left(\lambda_{1}, \ldots, \lambda_{r}\right) \vdash n$, let
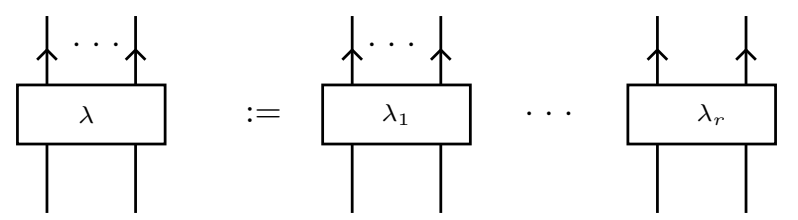

then we define

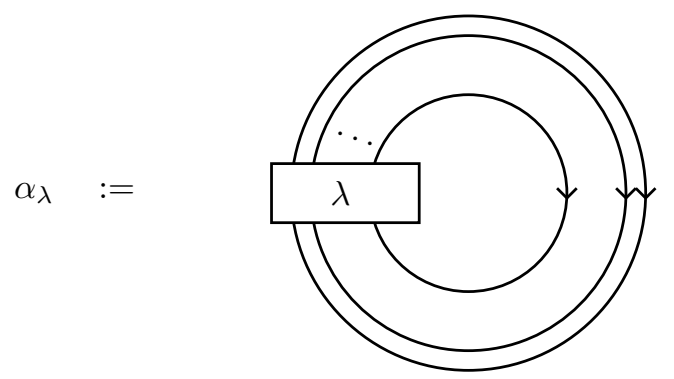

with $\alpha_{k}:=\alpha_{(k)}$.

Lemma 4.4 below shows that we could replace the permutation in (39) by the image under $\mathcal{T}_{n}$ of any $g \in S_{n}$ such that $\operatorname{sh}(g)=\lambda$. We choose (39) because it will be convenient for later calculations.

Lemma 4.4. Suppose that $g_{1}, g_{2} \in S_{n}$ are conjugate, so that $\operatorname{sh}\left(g_{1}\right)=\operatorname{sh}\left(g_{2}\right)$. Then

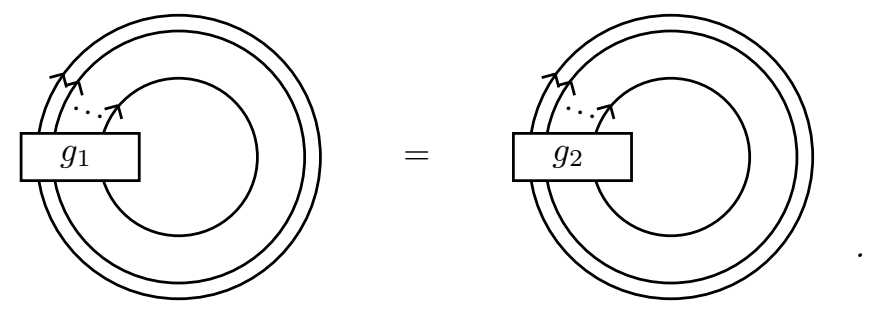


Proof. We use the fact that $g_{1}=h^{-1} g_{2} h$ for some $h \in S_{n}$. Replacing $g_{1}$ by $h^{-1} g_{2} h$, we slide $h$ around the closed diagram to cancel it with $h^{-1}$,

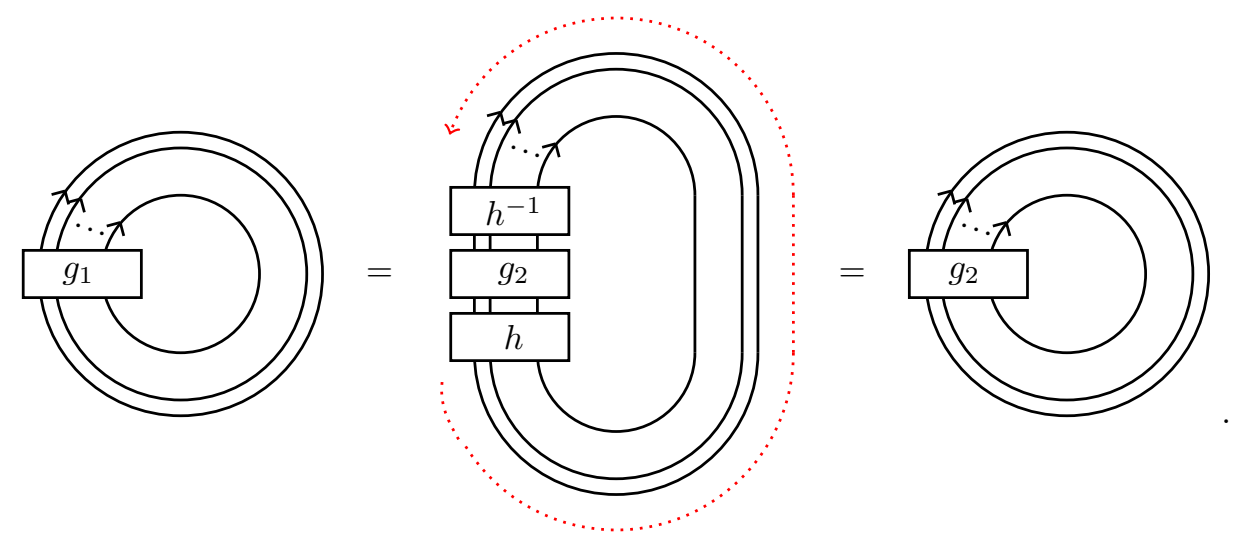

REMARK 4.5. In [13] Khovanov also studies $\mathcal{H}$, the Karoubi envelope (or idempotent completion) of $\mathcal{H}^{\prime}$. It is $\mathcal{H}$ which conjecturally categorifies the Heisenberg algebra. Since taking the Karoubi envelope of a category does not change its center, all the results we prove about $\operatorname{End}_{\mathcal{H}^{\prime}}(\mathbb{1})$ also hold for $\operatorname{End}_{\mathcal{H}}(\mathbb{1})$.

4.2. Diagrams AS BIMOdUle homomorphisms. In order to establish an isomorphism between $\operatorname{End}_{\mathcal{H}^{\prime}}(\mathbb{1})$ and $\Lambda^{*}$, we will make use of a family of representations of the monoidal category $\mathcal{H}^{\prime}$ constructed in [13].

Recall that for algebras $A$ and $B, M$ is an $(A, B)$-bimodule if it is a left $A$-module and a right $B$-module, and the actions of $A$ and $B$ are compatible. To describe the representations $\mathcal{H}^{\prime}$, we start by setting some notation for $\left(\mathbb{C}\left[S_{k_{1}}\right], \mathbb{C}\left[S_{k_{2}}\right]\right)$-bimodules. All inclusions are assumed to be the standard ones $\iota_{k, n}: \mathbb{C}\left[S_{k}\right] \rightarrow \mathbb{C}\left[S_{n}\right]$ introduced in Section 2. Suppose that $k_{1}, k_{2} \leqslant n$. We write:

- $(n)$ for $\mathbb{C}\left[S_{n}\right]$ considered as a $\left(\mathbb{C}\left[S_{n}\right], \mathbb{C}\left[S_{n}\right]\right)$-bimodule.

- $(n)_{k_{2}}$ for $\mathbb{C}\left[S_{n}\right]$ considered as a $\left(\mathbb{C}\left[S_{n}\right], \mathbb{C}\left[S_{k_{2}}\right]\right)$-bimodule.

- $k_{1}(n)$ for $\mathbb{C}\left[S_{n}\right]$ considered as a $\left(\mathbb{C}\left[S_{k_{1}}\right], \mathbb{C}\left[S_{n}\right]\right)$-bimodule.

- ${ }_{k_{1}}(n)_{k_{2}}$ for $\mathbb{C}\left[S_{n}\right]$ considered as a $\left(\mathbb{C}\left[S_{k_{1}}\right], \mathbb{C}\left[S_{k_{2}}\right]\right)$-bimodule.

Let $\mathcal{S}^{\prime}$ be the category whose objects are compositions of induction and restriction functors of symmetric groups. We write

$$
\operatorname{Ind}_{n}^{n+1}:=\operatorname{Ind}_{S_{n}}^{S_{n+1}} \quad \text { and } \quad \operatorname{Res}_{n}^{n+1}:=\operatorname{Res}_{S_{n}}^{S_{n+1}} .
$$

Since induction from $S_{n}$ to $S_{n+1}$ is given by tensoring on the left by $(n+1)_{n}$ and restriction from $S_{n+1}$ to $S_{n}$ is given by tensoring on the left by ${ }_{n}(n+1)$, the objects in $\mathcal{S}^{\prime}$ can be reinterpreted as $\left(\mathbb{C}\left[S_{k_{1}}\right], \mathbb{C}\left[S_{k_{2}}\right]\right)$-bimodules for $k_{1}, k_{2} \geqslant 0$. Most of our calculations will use this interpretation.

EXAMPLE 4.6. One object in $\mathcal{S}^{\prime}$ is the composition

$$
\operatorname{Res}_{4}^{5} \circ \operatorname{Ind}_{4}^{5} \circ \operatorname{Ind}_{3}^{4} \circ \operatorname{Res}_{3}^{4} .
$$

In the language of bimodules, this is the $\left(\mathbb{C}\left[S_{4}\right], \mathbb{C}\left[S_{4}\right]\right)$-bimodule

$$
{ }_{4}(5)_{4}(4)_{3}(4) \text {. }
$$

The morphisms in $\mathcal{S}^{\prime}$ are certain natural transformations of these compositions (or, equivalentely, certain bimodule homomorphisms). Like $\mathcal{H}^{\prime}$, morphisms in $\mathcal{S}^{\prime}$ can be presented diagrammatically as oriented compact 1-manifolds immersed in $\mathbb{R} \times[0,1]$. 
Unlike $\mathcal{H}^{\prime}$, in $\mathcal{S}^{\prime}$ we label the regions of the strip $\mathbb{R} \times[0,1]$ by non-negative integers, so that if there is an upwards oriented line separating two regions and the right region is labeled by $n$, then the left region must be labeled by $n+1$. The diagram

$$
n+1 \uparrow n
$$

denotes the identity endomorphism of the induction functor $\operatorname{Ind}_{n}^{n+1}$ or alternatively the identity endomorphism of the bimodule $(n+1)_{n}$.

If there is a downward oriented line separating two regions and the right is labeled by $n+1$ then the left must be labeled by $n$. The diagram

$$
n \downarrow n+1
$$

denotes the identity endomorphism of the restriction functor $\operatorname{Res}_{n}^{n+1}$ or alternatively the identity endomorphism of the bimodule ${ }_{n}(n+1)$.

The bimodule maps associated to the four cups and caps are:
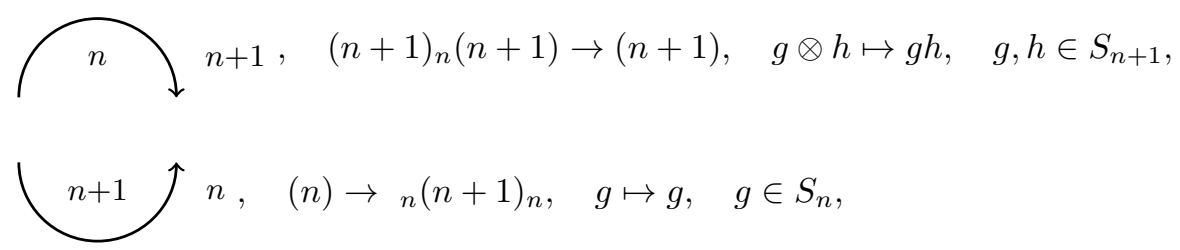

$$
{ }_{n+1} n, \quad{ }_{n}(n+1)_{n} \rightarrow(n), \quad g \mapsto \operatorname{pr}_{n}(g)= \begin{cases}g & g(n+1)=n+1 \\ 0 & \text { otherwise }\end{cases}
$$

$$
\uparrow n \int n+1,(n+1) \rightarrow(n+1)_{n}(n+1),
$$

where (44) is determined by the condition that

$$
1_{n+1} \mapsto \sum_{i=1}^{n+1} s_{i} s_{i+1} \cdots s_{n} \otimes s_{n} \cdots s_{i+1} s_{i}=\sum_{g \in \mathcal{L C}_{n}^{n+1}} g \otimes g^{-1} .
$$

Finally, the upward crossing is the bimodule map

$$
\searrow^{\searrow}, \quad(n+2)_{n} \rightarrow(n+2)_{n}, \quad g \mapsto g s_{n+1}, \quad g \in S_{n+2} .
$$

Any diagram that has a region labeled with a negative number is set to $\mathbf{0}$. It is shown in [13] that all diagrams are compatible with isotopy.

REMARK 4.7. Closed diagrams in $\mathcal{S}^{\prime}$ with outside region labeled by $n$ correspond to $\left(\mathbb{C}\left[S_{n}\right], \mathbb{C}\left[S_{n}\right]\right)$-bimodule endomorphisms of $(n)$. The algebra of such bimodule endomorphisms is isomorphic to $Z\left(\mathbb{C}\left[S_{n}\right]\right)$ via the map which sends $f \in \operatorname{End}_{\left(\mathbb{C}\left[S_{n}\right], \mathbb{C}\left[S_{n}\right]\right)}\left(\mathbb{C}\left[S_{n}\right]\right)$ to $f\left(1_{n}\right)$. In this way closed diagrams in $\mathcal{S}^{\prime}$ may be regarded as elements of the center of the appropriate group algebra.

Khovanov shows that the diagrams in $\mathcal{S}^{\prime}$ satisfy the defining relations for morphisms in $\mathcal{H}^{\prime}$. As a result, given an endomorphism of $\mathcal{H}^{\prime}$, after labeling the far right region by a non-negative integer, one obtains a well-defined bimodule homomorphism in $\mathcal{S}^{\prime}$. For example, using the bimodule definitions of cups and caps (41)-(44) we can 
compute that the clockwise-oriented curl generator $c_{0}$ maps to multiplication by $n+1$ when we label the outside region with $n+1$ :

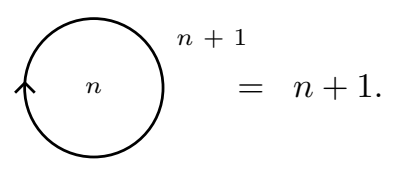

In other words, the endomorphism $c_{0} \in \operatorname{End}_{\mathcal{H}^{\prime}}(\mathbb{1})$ becomes the scalar $n+1$ in $Z\left(\mathbb{C}\left[S_{n+1}\right]\right)$.

$\mathcal{S}^{\prime}$ is the direct sum of categories

$$
\mathcal{S}^{\prime}=\bigoplus_{k=0}^{\infty} \mathcal{S}_{k}^{\prime}
$$

where $\mathcal{S}_{k}^{\prime}$ contains all objects such that induction or restriction starts at $S_{k}$ (i.e. the rightmost region of the diagram is labeled by $k$ ). There are functors $f_{k}^{\mathcal{H}^{\prime}}: \mathcal{H}^{\prime} \rightarrow \mathcal{S}_{k}^{\prime}$ such that the object $\epsilon_{1} \epsilon_{2} \ldots \epsilon_{n}$ is taken to a composition of induction and restriction functors with + sent to $\operatorname{Ind}_{i}^{i+1}$ and - sent to $\operatorname{Res}_{i-1}^{i}$ where $i$ in each case is determined by the requirement that induction/restriction begin from $S_{k} \cdot f_{k}^{\mathcal{H}^{\prime}}$ takes a diagram from $\mathcal{H}^{\prime}$ to $\mathcal{S}_{k}^{\prime}$ by labeling regions so that the rightmost region is labeled with a $k$ and then interpreting the diagram as an element of $\mathcal{S}_{k}^{\prime}$.

EXAMPLE 4.8. $f_{5}^{\mathcal{H}^{\prime}}: \mathcal{H}^{\prime} \rightarrow \mathcal{S}_{5}^{\prime}$ takes

$$
\begin{aligned}
(++-+-) & \stackrel{f_{5}^{\mathcal{H}^{\prime}}}{\longmapsto} \operatorname{Ind}_{5}^{6} \circ \operatorname{Ind}_{4}^{5} \circ \operatorname{Res}_{4}^{5} \circ \operatorname{Ind}_{4}^{5} \circ \operatorname{Res}_{4}^{5}, \\
(-++) & \stackrel{f_{5}^{\mathcal{H}^{\prime}}}{\longmapsto} \operatorname{Res}_{6}^{7} \operatorname{Ind}_{6}^{7} \operatorname{Ind}_{5}^{6} .
\end{aligned}
$$

In the remainder of this section we calculate the image of a number of important diagrams in $\mathcal{H}^{\prime}$ under the functors $f_{k}^{\mathcal{H}^{\prime}}$.

LEMma 4.9 ([13, Section 4]). The diagram

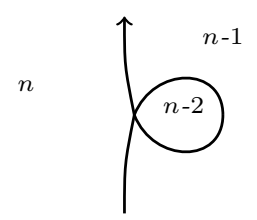

is the endomorphism of $(n)_{n-1}$ which is right multiplication by $J_{n}$.

Proof. The right twist curl can be written as the composition of a cup, a crossing, and a cap.

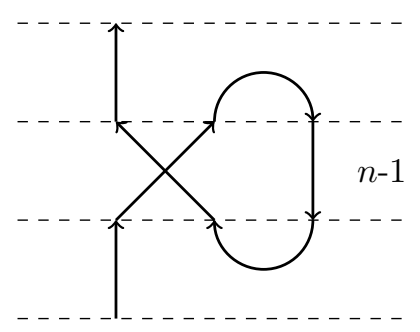


Applying the endomorphism to $1_{n}$ gives

$$
\begin{aligned}
1_{n} & \mapsto \sum_{i=1}^{n-1} s_{i} \cdots s_{n-2} \otimes s_{n-2} \cdots s_{i} \mapsto \sum_{i=1}^{n-1} s_{i} \cdots s_{n-2} s_{n-1} \otimes s_{n-2} \cdots s_{i} \\
& \mapsto \sum_{i=1}^{n-1} s_{i} \cdots s_{n-2} s_{n-1} s_{n-2} \cdots s_{i}=J_{n}
\end{aligned}
$$

where the equality holds by (8).

LEMma 4.10. Let $k \leqslant n$ :

(1) The diagram

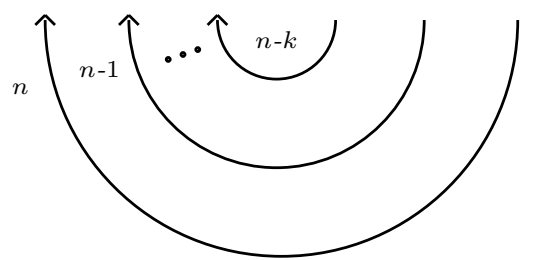

corresponds to the bimodule homomorphism $(n) \rightarrow(n)_{n-k}(n)$ which sends

$$
1_{n} \mapsto \sum_{g \in \mathcal{L C}_{n-k}^{n}} g \otimes g^{-1} .
$$

(2) Let $\mu \vdash k$ and $x_{1}, x_{2} \in(n)$. The diagram

$n$

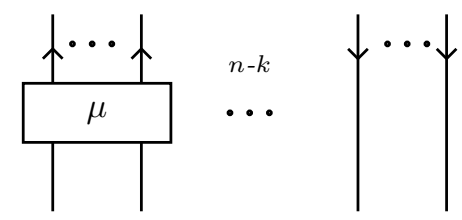

corresponds to the bimodule homomorphism $(n)_{n-k}(n) \rightarrow(n)_{n-k}(n)$ which sends

$$
x_{1} \otimes x_{2} \mapsto x_{1} \sigma_{\mu, n} \otimes x_{2} .
$$

Proof.

(1) We can factor the diagram in part (1) of the lemma into a vertically stacked composition of $k\left(S_{n}, S_{n}\right)$-bimodule homomorphisms which we label $f_{1}, f_{2}$, $\ldots, f_{k}$ on the right below
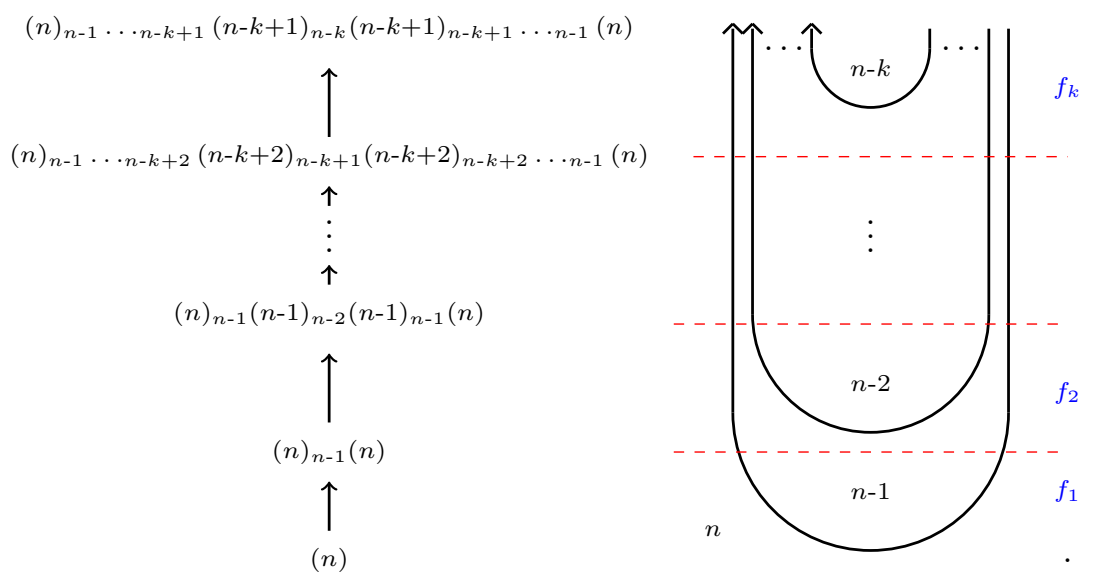
The homomorphism corresponding to $f_{1}$ sends

$$
1_{n} \stackrel{f_{1}}{\longmapsto} \sum_{g \in \mathcal{L C}_{n-1}^{n}} g \otimes g^{-1}
$$

and for $2 \leqslant i \leqslant k$, and $x^{(j)}, y^{(j)} \in \mathbb{C}\left[S_{j}\right]$, the homomorphism corresponding to $f_{i}$ sends

$$
\begin{aligned}
x^{(n)} & \otimes \cdots \otimes x^{(n-i+2)} \otimes y^{(n-i+2)} \otimes \cdots \otimes y^{(n)} \\
& \mapsto \sum_{g \in \mathcal{L C}_{n-i}^{n-i+1}} x^{(n)} \otimes \cdots \otimes x^{(n-i+2)} \otimes g \otimes g^{-1} \otimes y^{(n-i+2)} \otimes \cdots \otimes y^{(n)}
\end{aligned}
$$

Noting that the final $\left(S_{n}, S_{n}\right)$-bimodule in the composition above is isomorphic to $(n)_{n-k}(n)$ by the map which sends

$$
\begin{aligned}
x^{(n)} \otimes \cdots \otimes x^{(n-k+1)} \otimes y^{(n-k+1)} \otimes \cdots & \otimes y^{(n)} \\
& \mapsto x^{(n)} \ldots x^{(n-k+1)} \otimes y^{(n-k+1)} \ldots y^{(n)}
\end{aligned}
$$

we get that the composition of $f_{1}, \ldots, f_{k}$ sends

$$
\begin{array}{r}
1_{n} \mapsto \sum_{g^{(n)} \in \mathcal{L C}_{n-1}^{n}} \ldots \sum_{g^{(n-k+1)} \in \mathcal{L C}_{n-k}^{n-k+1}} g^{(n)} \ldots g^{(n-k+1)} \otimes\left(g^{(n-k+1)}\right)^{-1} \ldots\left(g^{(n)}\right)^{-1} \\
=\sum_{g \in \mathcal{L C}_{n-k}^{n}} g \otimes g^{-1} .
\end{array}
$$

(2) For simplicity we let $\mu=(k)$. The calculation for arbitrary $\mu$ easily generalizes from this case. As in part (1) of the proof, we factor the diagram in part (2) into a vertically stacked composition of diagrams

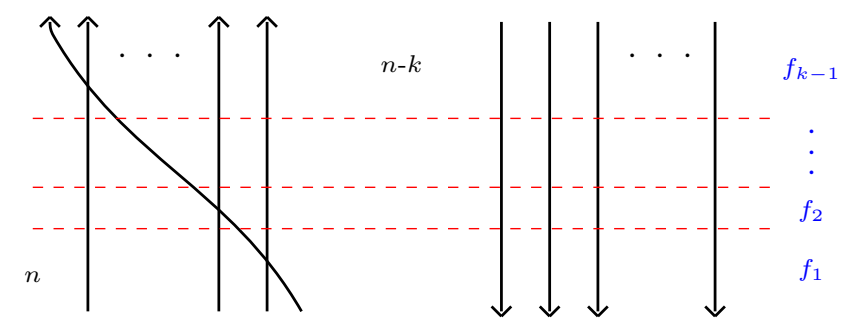

with corresponding $\left(S_{n}, S_{n}\right)$-bimodule endomorphisms of $(n)_{n-k}(n)$ labeled by $f_{1}, f_{2}, \ldots, f_{k-1}$. In particular, reading left to right the diagram for $f_{i}$ consists of $k$ upward pointing strands on the left with the $(k-i)$ th strand and $(k-i+1)$ th strand crossed, and $k$ downward pointing strands on the right. The region to the right of the crossing is labeled by $n-k+i-1$, the regions above and below are labeled by $n-k+i$, and the region to the left is labeled by $n-k+i+1$. In order to use (45) to apply $f_{i}$ to element $x_{1}^{\prime} \otimes x_{2}^{\prime} \in(n)_{n-k}(n)$, we use the isomorphism

$$
\begin{aligned}
(n)_{n-k}(n) & \stackrel{\sim}{\rightarrow}(n)_{n-k+i+1}(n-k+i+1)_{n-k+i-1}(n-k+i-1)_{n-k}(n) \\
x_{1}^{\prime} \otimes x_{2}^{\prime} & \mapsto \quad x_{1}^{\prime} \otimes 1_{n-k+i+1} \otimes 1_{n-k+i-1} \otimes x_{2}^{\prime} .
\end{aligned}
$$

Applying $f_{i}$ via (45) gives

$$
x_{1}^{\prime} \otimes 1_{n-k+i+1} \otimes 1_{n-k+i-1} \otimes x_{2}^{\prime} \stackrel{f_{i}}{\longmapsto} x_{1}^{\prime} \otimes s_{n-k+i} \otimes 1_{n-k+i-1} \otimes x_{2}^{\prime}
$$


and using the inverse of (47) we obtain $x_{1}^{\prime} s_{n-k+i} \otimes x_{2}^{\prime} \in(n)_{n-k}(n)$. Starting with $x_{1} \otimes x_{2} \in(n)_{n-k}(n)$ and using this procedure we calculate

$$
\begin{aligned}
f_{k-1} \circ \cdots \circ f_{2} \circ f_{1}\left(x_{1} \otimes x_{2}\right) & =x_{1} s_{n-k+1} s_{n-k+2} \ldots s_{n-1} \otimes x_{2} \\
& =x_{1} \sigma_{(k), n} \otimes x_{2} .
\end{aligned}
$$

Lemma 4.11. As elements of $Z\left(\mathbb{C}\left[S_{n}\right]\right)$ :

(1) $f_{n}^{\mathcal{H}^{\prime}}\left(c_{k}\right)=\sum_{i=1}^{n} s_{i} \cdots s_{n-1} J_{n}^{k} s_{n-1} \cdots s_{i}$,

(2) $f_{n}^{\mathcal{H}^{\prime}}\left(\tilde{c}_{k}\right)=\operatorname{pr}_{n}\left(J_{n+1}^{k}\right)$.

(3) $f_{n}^{\mathcal{H}^{\prime}}\left(\alpha_{\mu}\right)= \begin{cases}A_{\mu, n} & \text { if }|\mu| \leqslant n \\ 0 & \text { otherwise. }\end{cases}$

Proof. (1)-(2) are found in [13, Section 4] and can be computed from the definitions of cups and caps and Lemma 4.9. To compute (3), we can decompose the diagram for $\alpha_{\mu}$ into three vertically stacked diagrams

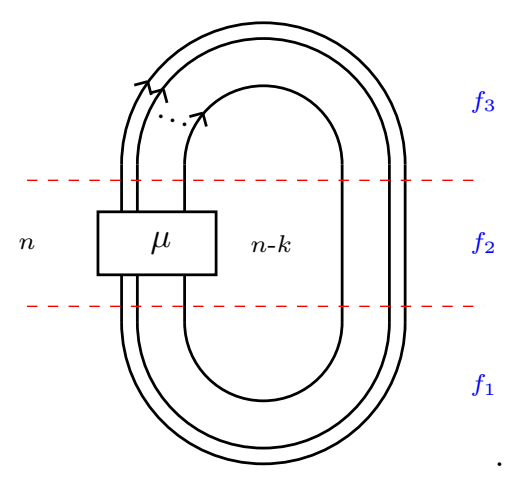

Using Lemma 4.10 we see that the homomorphisms $f_{1}$ and $f_{2}$ map

$$
1_{n} \stackrel{f_{1}}{\longmapsto} \sum_{g \in \mathcal{L \mathcal { C } _ { n - k } ^ { n }}} g \otimes g^{-1} \stackrel{f_{2}}{\longmapsto} \sum_{g \in \mathcal{\mathcal { C } _ { n - k } ^ { n }}} g \sigma_{\mu, n} \otimes g^{-1} .
$$

Then (41) implies that $f_{3}$ sends

$$
\sum_{g \in \mathcal{L} \mathcal{C}_{n-k}^{n}} g \sigma_{\mu, n} \otimes g^{-1} \stackrel{f_{3}}{\longmapsto} \sum_{g \in \mathcal{L} \mathcal{C}_{n-k}^{n}} g \sigma_{\mu, n} g^{-1}=A_{\mu, n} .
$$

\section{The ISOMORPhism $\varphi: \operatorname{End}_{\mathcal{H}^{\prime}}(\mathbb{1}) \longrightarrow \Lambda^{*}$}

In this section we establish the algebra isomorphism $\operatorname{End}_{\mathcal{H}^{\prime}}(\mathbb{1}) \cong \Lambda^{*}$. The proof is somewhat analogous to Ivanov and Kerov's proof of a related isomorphism connecting shifted symmetric functions to the representation theory of symmetric groups (see [8, Theorem 9.1]).

In [13, Section 4], Khovanov defines a grading on $\operatorname{End}_{\mathcal{H}^{\prime}}(\mathbb{1})$ by setting

$$
\operatorname{deg}\left(c_{0}\right):=0, \quad \text { and } \quad \operatorname{deg}\left(c_{k}\right):=k+1, \quad \text { for } k \geqslant 1 .
$$

We will consider the increasing filtration induced by this grading. A relationship between the elements $\left\{c_{k}\right\}_{k \geqslant 0}$ and $\left\{\alpha_{k}\right\}_{k \geqslant 1}$ is then given in terms of this filtration as follows.

Proposition 5.1. For any $k \geqslant 1$,

$$
\alpha_{k}=c_{k-1}+\text { l.o.t. }
$$


Proof. This follows from repeated application of the dot sliding moves (33)-(34) and bubble sliding move (35). Notice that with each application of these moves, we get a single term from the same filtered part plus additional terms of lower degree.

Since the elements $c_{0}, c_{1}, \ldots$ are algebraically independent generators of $\operatorname{End}_{\mathcal{H}^{\prime}}(\mathbb{1})$, we immediately obtain the following.

COROLLARY 5.2. The elements $\alpha_{1}, \alpha_{2}, \ldots$ are algebraically independent generators of $\operatorname{End}_{\mathcal{H}^{\prime}}(\mathbb{1})$.

For any $\lambda \vdash n$, composing $f_{n}^{\mathcal{H}^{\prime}}$ with the normalized character $\widetilde{\chi}^{\lambda}$ gives a map

$$
\left(\tilde{\chi}^{\lambda} \circ f_{n}^{\mathcal{H}^{\prime}}\right): \operatorname{End}_{\mathcal{H}^{\prime}}(\mathbb{1}) \rightarrow \mathbb{C}
$$

and allows us to define a homomorphism $\varphi: \operatorname{End}_{\mathcal{H}^{\prime}}(\mathbb{1}) \rightarrow \operatorname{Fun}(\mathcal{P}, \mathbb{C})$. Specifically, for $x \in \operatorname{End}_{\mathcal{H}^{\prime}}(\mathbb{1})$, we write

$$
[\varphi(x)](\lambda):=\left(\widetilde{\chi}^{\lambda} \circ f_{n}^{\mathcal{H}^{\prime}}\right)(x) .
$$

Combining Lemma 4.11.3 with (7) implies that for $\mu \vdash k$

$$
\left[\varphi\left(\alpha_{\mu}\right)\right](\lambda)= \begin{cases}\frac{(n\lfloor k)}{\operatorname{dim} L^{\lambda}} \chi^{\lambda}\left(\phi_{k, n}(\mu)\right) & \text { if } k \leqslant n \\ 0 & \text { otherwise. }\end{cases}
$$

THEOREM 5.3. The map $\varphi$ induces an algebra isomorphism End $_{\mathcal{H}^{\prime}}(\mathbb{1}) \rightarrow \Lambda^{*} \subseteq$ $\operatorname{Fun}(\mathcal{P}, \mathbb{C})$ with

$$
\alpha_{\mu} \stackrel{\varphi}{\longmapsto} p_{\mu}^{\#}
$$

Proof. Let $\lambda \vdash n . \varphi$ is an algebra homomorphism because $f_{n}^{\mathcal{H}^{\prime}}$ is a homomorphism from $\operatorname{End}_{\mathcal{H}^{\prime}}(\mathbb{1})$ to $Z\left(\mathbb{C}\left[S_{n}\right]\right)$ and $\tilde{\chi}^{\lambda}$ is a homomorphism when restricted to $Z\left(\mathbb{C}\left[S_{n}\right]\right)$. By Proposition 3.5 and (49), $\alpha_{\mu}$ maps to $p_{\mu}^{\#}$. Since the $\left\{p_{k}^{\#}\right\}_{k \geqslant 1}$ (respectively $\left\{\alpha_{k}\right\}_{k \geqslant 1}$ ) are algebraically independent generators of $\Lambda^{*}\left(\operatorname{resp} . \operatorname{End}_{\mathcal{H}^{\prime}}(\mathbb{1})\right), \varphi$ must be an isomorphism.

Note that Theorem 5.3 along with Lemma 4.4 imply that when $\mu \vdash n$,

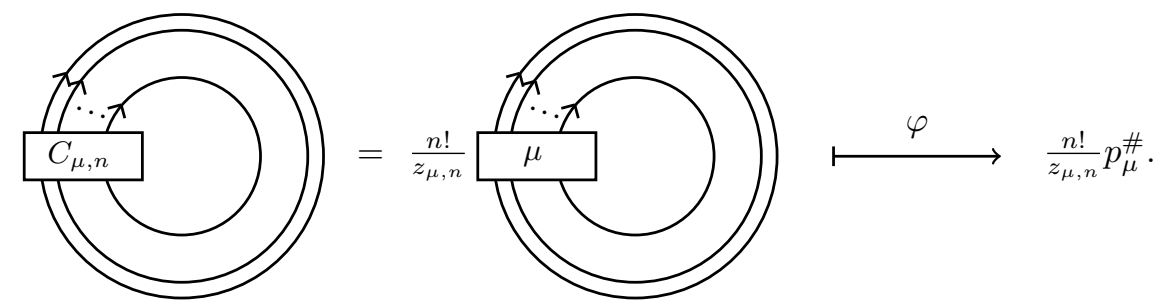

For $\lambda \vdash n$ recall that $E_{\lambda}$ is the Young idempotent associated to $\lambda$.

THEOREM 5.4. The isomorphism $\varphi$ sends

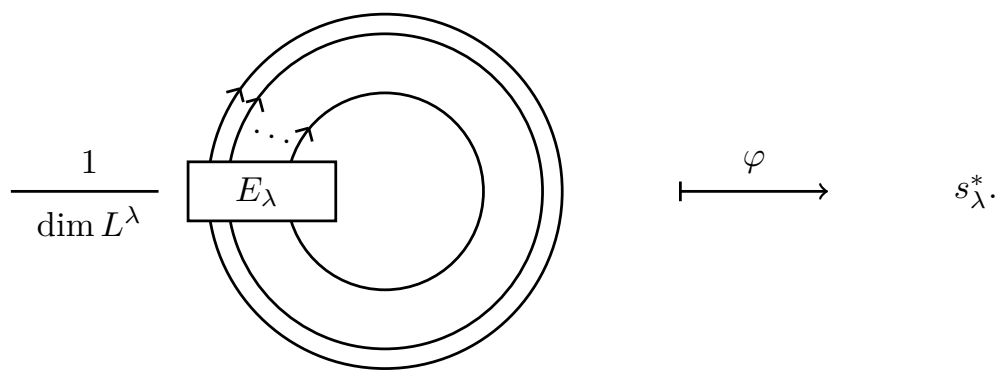


Proof. Recall that

$$
\left(\frac{1}{\operatorname{dim} L^{\lambda}}\right) E_{\lambda}=\sum_{\mu \vdash n} \frac{\chi^{\lambda}(\mu)}{n !} C_{\mu, n}
$$

(see for example [16]), while

$$
s_{\lambda}^{*}=\sum_{\mu \vdash n} \frac{\chi^{\lambda}(\mu)}{z_{\mu, n}} p_{\mu}^{\#} .
$$

The result then follows from (50).

The previous theorems gave graphical realizations of some important bases of $\Lambda^{*}$. Now we go the other way, and describe Khovanov's curl generators $\tilde{c}_{k}$ and $c_{k}$ as elements of $\Lambda^{*}$. It is this description that makes an explicit connection between $\mathcal{H}^{\prime}$ and the transition and co-transition measures of Kerov.

THEOREM 5.5. The isomorphism $\varphi$ sends:

(1) $\tilde{c}_{k} \mapsto \hat{m}_{k} \in \Lambda^{*}$

(2) $c_{k} \mapsto p_{1}^{\#} \check{m}_{k}=\widehat{b}_{k+2} \in \Lambda^{*}$.

Proof. Let $\lambda \vdash n$, then from Lemma 4.11 and Proposition 2.14 we have

$$
\left[\varphi\left(\tilde{c}_{k}\right)\right](\lambda)=\tilde{\chi}^{\lambda}\left(\operatorname{pr}_{n}\left(J_{n+1}^{k}\right)\right)=\hat{m}_{k}(\lambda)
$$

and

$$
\left[\varphi\left(c_{k}\right)\right](\lambda)=\tilde{\chi}^{\lambda}\left(\sum_{i=1}^{n} s_{i} \cdots s_{n-1} J_{n}^{k} s_{n-1} \cdots s_{i}\right)=p_{1}^{\#}(\lambda) \check{m}_{k}(\lambda)=\widehat{b}_{k+2}(\lambda) .
$$

REMARK 5.6. In [5], Farahat and Higman used the inductive structure of symmetric groups to construct a $\mathbb{C}$-algebra known as the Farahat-Higman algebra $\mathcal{K}_{\mathbb{C}}$ (see also [16, Example 24, Section I.7]). It follows from, for example [8], that there is an algebra isomorphism $\mathcal{K}_{\mathbb{C}} \cong \Lambda^{*}$, and the functors $f_{n}^{\mathcal{H}^{\prime}}$ can also be used to give a direct isomorphism between $\operatorname{End}_{\mathcal{H}^{\prime}}(\mathbb{1})$ and $\mathcal{K}_{\mathbb{C}}$. So in principle all of the appearances of shifted symmetric functions in the previous sections could be rephrased in the language of the Farahat-Higman algebra.

REMARK 5.7. Theorem 5.5 and Remark 3.8 together imply that the recursive relationships for $\left\{\hat{m}_{k}\right\}$ and $\left\{\hat{b}_{k}\right\}$ in Remark 2.13 and $\left\{c_{k}\right\}$ and $\left\{\tilde{c}_{k}\right\}$ in Lemma 4.3 are both consequences of the well-known relationship between the elementary and homogeneous symmetric functions:

$$
\sum_{i=0}^{k}(-1)^{i} e_{i} h_{n-i}=0
$$

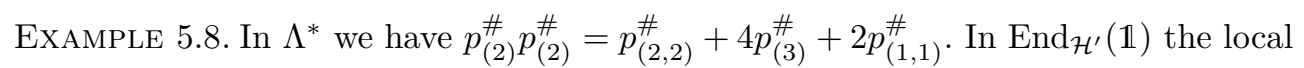
relations can be used to compute the corresponding equation:

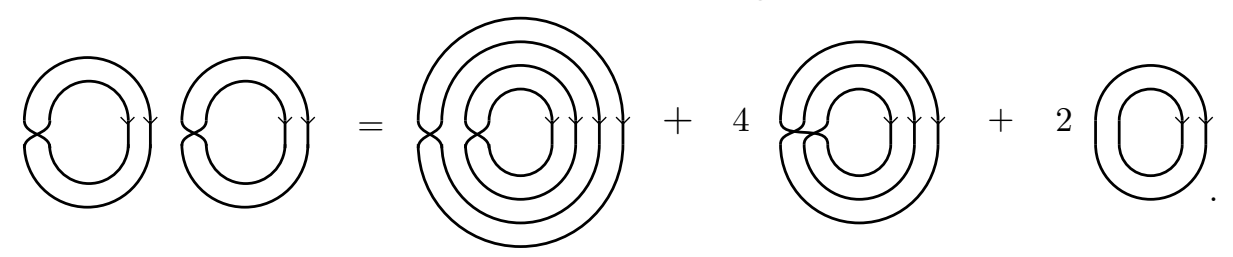


5.1. Involutions ON $\operatorname{End}_{\mathcal{H}^{\prime}}(\mathbb{1})$. In [13], Khovanov introduced three involutive autoequivalences on $\mathcal{H}^{\prime}$. Only one of these, which we denote as $\xi$, acts nontrivially on $\operatorname{End}_{\mathcal{H}^{\prime}}(\mathbb{1})$ where it gives an involutive algebra automorphism. For $D \in \operatorname{Hom}_{\mathcal{H}^{\prime}}\left(Q_{\epsilon_{1}}, Q_{\epsilon_{2}}\right), \xi$ is defined so that

$$
\xi(D):=(-1)^{c(D)} D
$$

where $c(D)$ is the total number of dots and crossings in the diagram. Thus, in $\operatorname{End}_{\mathcal{H}^{\prime}}(\mathbb{1}):$

$$
\begin{aligned}
& c_{k} \stackrel{\xi}{\longmapsto}(-1)^{k} c_{k}, \\
& \tilde{c}_{k} \stackrel{\xi}{\longmapsto}(-1)^{k} \tilde{c}_{k}, \\
& \alpha_{k} \stackrel{\xi}{\longmapsto}(-1)^{k-1} \alpha_{k} .
\end{aligned}
$$

In Section 4 of [18], Okounkov and Olshanski identified an involutive algebra automorphism $I: \Lambda^{*} \rightarrow \Lambda^{*}$ which acts on $f \in \Lambda^{*}$ such that for $\lambda \in \mathcal{P}$,

$$
[I(f)](\lambda)=f\left(\lambda^{\prime}\right),
$$

where $\lambda^{\prime}$ is the conjugate partition to $\lambda$. In particular

$$
\begin{aligned}
I\left(s_{\lambda}^{*}\right) & =s_{\lambda^{\prime}}^{*}, \\
I\left(e_{k}^{*}\right) & =h_{k}^{*}, \\
I\left(p_{k}^{\#}\right) & =(-1)^{k-1} p_{k}^{\#} .
\end{aligned}
$$

Proposition 5.9. The involution $\xi$ on $\operatorname{End}_{\mathcal{H}^{\prime}}(\mathbb{1})$ coincides with the involution $I$ on $\Lambda^{*}$.

Proof. This follows from the fact that $\left\{\alpha_{k}\right\}_{k \geqslant 1}$, (respectively $\left\{p_{k}^{\#}\right\}_{k \geqslant 1}$ ) generate $\operatorname{End}_{\mathcal{H}^{\prime}}(\mathbb{1})\left(\right.$ resp. $\left.\Lambda^{*}\right), \varphi\left(\alpha_{k}\right)=p_{k}^{\#}$, and a comparison of (53) and (56).

5.2. A graphical construction of the aCtion of $W_{1+\infty}$ on $\Lambda^{*}$. In [4], the trace $\operatorname{Tr}\left(\mathcal{H}^{\prime}\right)$ (or zeroth Hochschild homology) of $\mathcal{H}^{\prime}$ is shown to be isomorphic as an algebra to a quotient of the $\mathrm{W}$-algebra $W_{1+\infty}$. Like the center $\operatorname{End}_{\mathcal{H}^{\prime}}(\mathbb{1})$, which is the algebra of closed planar diagrams, the trace $\operatorname{Tr}\left(\mathcal{H}^{\prime}\right)$ has a purely graphical description, as the space of annular diagrams modulo Khovanov's local diagrammatic relations. More precisely, the underlying vector space of $\operatorname{Tr}\left(\mathcal{H}^{\prime}\right)$ is isomorphic to the span of annular diagrams, where an annular diagram $\tilde{f}$ is by definition a diagram obtained by taking an endomorphism $f \in \operatorname{End}_{\mathcal{H}^{\prime}}(X)$ for some object $X \in \mathcal{H}^{\prime}$, and closing it up to the right in an annulus. The multiplication in $\operatorname{Tr}\left(\mathcal{H}^{\prime}\right)$ is given by gluing annuli around one another:
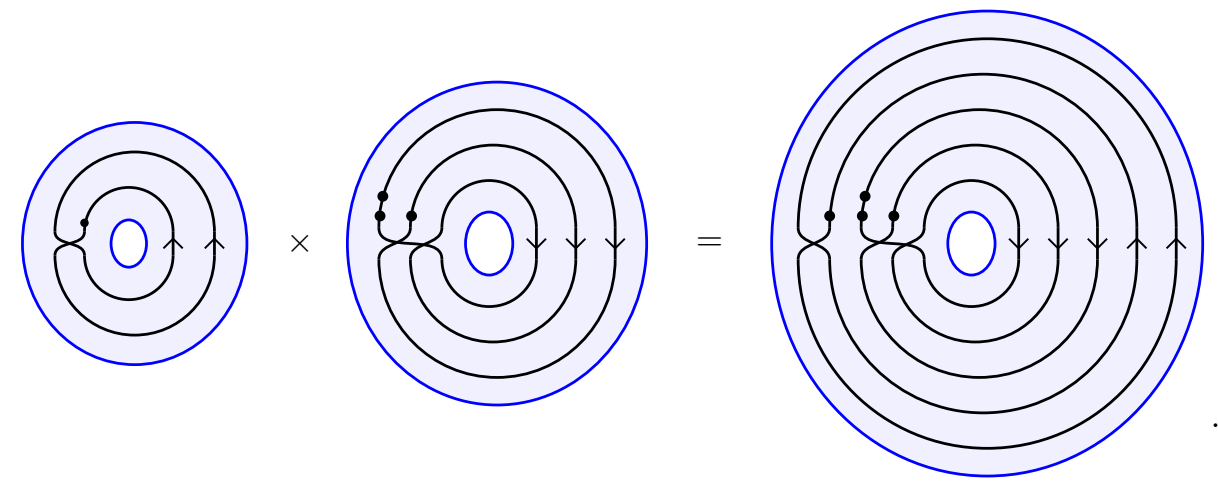
The action of $\operatorname{Tr}\left(\mathcal{H}^{\prime}\right)$ on $\operatorname{End}_{\mathcal{H}^{\prime}}(\mathbb{1})$ then acquires a graphical description: given an annular diagram $\tilde{f} \in \operatorname{Tr}\left(\mathcal{H}^{\prime}\right)$ and a closed planar diagram $g \in \operatorname{End}_{\mathcal{H}^{\prime}}(\mathbb{1})$, the closed planar diagram $\tilde{f} g \in \operatorname{End}_{\mathcal{H}^{\prime}}(\mathbb{1})$ is given by inserting a planar neighborhood of the closed diagram $g$ into the middle of the annulus:
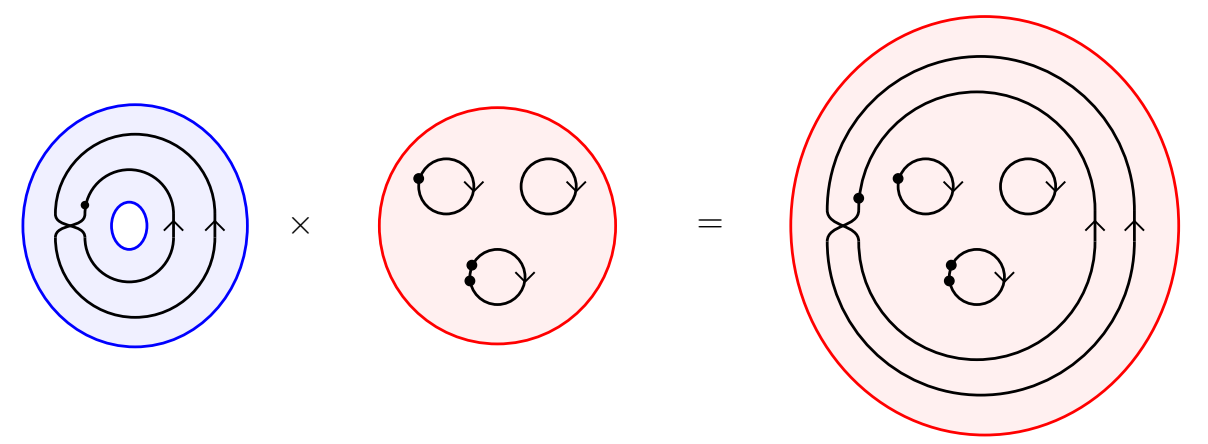

Thus, via the isomorphisms

$$
\operatorname{End}_{\mathcal{H}^{\prime}}(\mathbb{1}) \cong \Lambda^{*}, \quad \operatorname{Tr}\left(\mathcal{H}^{\prime}\right) \cong W_{1+\infty}
$$

of Theorem 5.3 and [4], respectively, we obtain a purely graphical construction of the action of $W_{1+\infty}$ on $\Lambda^{*}$. Such an action was first considered by Lascoux-Thibon in [14].

Acknowledgements. The authors would like to thank Ben Elias, Alexander Ellis, Sara Billey, Eugene Gorsky, Aaron Lauda, Carson Rogers, and Alistair Savage for helpful conversations. We would also like to thank Monica Vazirani for her valuable comments after a careful reading of an earlier draft of this paper. HK would like to thank Mikhail Khovanov for his suggestion to look for a relationship between the Heisenberg category and the combinatorics of symmetric functions.

\section{REFERENCES}

[1] Octavio Arizmendi, Takahiro Hasebe, Franz Lehner, and Carlos Vargas, Relations between cumulants in noncommutative probability, Adv. Math. 282 (2015), 56-92.

[2] Philippe Biane, Representations of symmetric groups and free probability, Adv. Math. 138 (1998), no. 1, 126-181.

[3] Characters of symmetric groups and free cumulants, in Asymptotic combinatorics with applications to mathematical physics (St. Petersburg, 2001), Lecture Notes in Mathematics, vol. 1815, Springer, 2003, pp. 185-200.

[4] Sabin Cautis, Aaron D. Lauda, Anthony M. Licata, and Joshua Sussan, W-algebras from Heisenberg categories, J. Inst. Math. Jussieu 17 (2017), no. 5, 981-1017.

[5] H. K. Farahat and Graham Higman, The centres of symmetric group rings, Proc. R. Soc. Lond., Ser. A 250 (1959), 212-221.

[6] Alice Guionnet, Vaughan F. R. Jones, and Dimitri Shlyakhtenko, Random matrices, free probability, planar algebras and subfactors, in Quanta of maths, Clay Mathematics Proceedings, vol. 11, American Mathematical Society, 2010, pp. 201-239.

[7] Akihito Hora and Nobuaki Obata, Quantum probability and spectral analysis of graphs, Theoretical and Mathematical Physics, Springer, 2007.

[8] Vladimir Ivanov and Sergei Kerov, The algebra of conjugacy classes in symmetric groups, and partial permutations, Zap. Nauchn. Semin. (POMI) 256 (1999), 95-120.

[9] Vladimir Ivanov and Grigori Olshanski, Kerov's central limit theorem for the Plancherel measure on Young diagrams, in Symmetric functions 2001: surveys of developments and perspectives, NATO Science Series II: Mathematics, Physics and Chemistry, vol. 74, Kluwer Academic Publishers, 2002, pp. 93-151.

[10] Sergei Kerov, Transition probabilities of continual Young diagrams and the Markov moment problem, Funkts. Anal. Prilozh. 27 (1993), no. 2, 32-49. 
[11] _ Anisotropic Young diagrams and symmetric Jack functions, Funkts. Anal. Prilozh. 34 (2000), no. 1, 51-64.

[12] Sergei Kerov and Grigori Olshanski, Polynomial functions on the set of Young diagrams, C. R. Math. Acad. Sci. Paris 319 (1994), no. 2, 121-126.

[13] Mikhail Khovanov, Heisenberg algebra and a graphical calculus, Fundam. Math. 225 (2014), no. $1,169-210$.

[14] Alain Lascoux and Jean-Yves Thibon, Vertex operators and the class algebras of symmetric groups, Zap. Nauchn. Semin. (POMI) 283 (2001), 156-177.

[15] Michel Lassalle, Jack polynomials and free cumulants, Adv. Math. 222 (2009), no. 6, 2227-2269.

[16] Ian G. Macdonald, Symmetric functions and Hall polynomials, second ed., Oxford Classic Texts in the Physical Sciences, Clarendon Press, 2015.

[17] Andrei Okounkov, Quantum immanants and higher Capelli identities, Transform. Groups 1 (1996), no. 1-2, 99-126.

[18] Andrei Okounkov and Grigori Olshanski, Shifted Schur functions, Algebra Anal. 9 (1997), no. 2, $73-146$.

[19] Roland Speicher and Reza Woroudi, Boolean convolution, in Free probability theory (Waterloo, ON, 1995), Fields Institute Communications, vol. 12, American Mathematical Society, 1997, pp. $267-279$.

[20] Richard P. Stanley, Enumerative combinatorics. Vol. 2, Cambridge Studies in Advanced Mathematics, vol. 62, Cambridge University Press, 1999.

[21] Anatoly Vershik and Andrei Okounkov, A new approach to representation theory of symmetric groups. II, Zap. Nauchn. Semin. (POMI) 307 (2004), 57-98.

Henry Kvinge, University of California Davis, Department of Mathematics, Davis, CA USA Colorado State University, Department of Mathematics, Fort Collins, CO USA

E-mail : henry.kvinge@colostate.edu

Anthony M. Licata, Mathematical Sciences Institute, Australian National University, Canberra, Australia

E-mail : amlicata@gmail.com

Stuart Mitchell, Mathematical Sciences Institute, Australian National University, Canberra, Australia

E-mail : stuart.a.mitchell@gmail.com 